\title{
Investigating Functional Performance and
}

Substituent Effect in Modelling Molecular Structure, UV-Visible Spectra and Optical Properties of D- $\pi-A$ Conjugated Organic Dye Molecules: A DFT and TDDFT Study.

\section{Garima Chanana}

GGSIPU: Guru Gobind Singh Indraprastha University

Kriti Batra ( $\nabla$ kriti.jpu@gmail.com )

GGS Indraprastha University

\section{Research Article}

Keywords: DFT, TD-DFT, Optical properties, UV-Visible Spectra, D-囚-A organic dye molecules, Disperse Red 1, Disperse Red 73

Posted Date: March 11th, 2021

DOl: https://doi.org/10.21203/rs.3.rs-249110/v1

License: (1) This work is licensed under a Creative Commons Attribution 4.0 International License. Read Full License

Version of Record: A version of this preprint was published at Journal of Molecular Modeling on July 23rd, 2021. See the published version at https://doi.org/10.1007/s00894-021-04824-y. 


\title{
Investigating functional performance and substituent effect in modelling Molecular Structure, UV-Visible Spectra and Opti- cal properties of $\mathrm{D}-\pi$-A conjugated organic dye molecules: A DFT and TD-DFT study.
}

\author{
Garima Chanana ${ }^{a}$ Kriti Batra ${ }^{a, *}$ \\ ${ }^{a}$ University School of Basic and Applied Sciences, GGS Indraprastha University, Dwarka, Delhi-110078, \\ India
}

\begin{abstract}
The molecular structure, UV-Visible spectra, and optical properties of D- $\pi$-A conjugated organic dye molecules (Disperse Red 1 (DR1) and Disperse Red 73 (DR73)) were analyzed using Density Functional Theory (DFT) and Time-Dependent Density Functional Theory (TD-DFT) and compared with Azobenzene molecule to study the effect of Donor and Acceptor substituents on the molecular properties. The performance of DFT functionals is investigated using B3LYP hybrid functional and three long-range corrected functionals (CAM-B3LYP, LC- $\omega$ PBE, and $\omega$ B97XD) in conjunction with 6-31G $(\mathrm{d}, \mathrm{p})$ basis set. Using TD-DFT, we calculate the vertical excitation energies and transition dipole moment values for 100 excited states. These values were further utilized to calculate frequency dependent polarizability under Sum-Over-States (SOS) formalism and refractive index of these molecular systems. We observe that B3LYP and CAM-B3LYP perform well in estimating molecular structures while CAM-B3LYP models the UV-Visible spectra of molecules with the least error compared to experimental results. Therefore, CAM-B3LYP is reported to be the most suitable candidate for modelling disperse dye molecules. Large polarizability response is also observed for these molecules (DR1 and DR73) in comparison to parent Azobenzene structure due to charge transfer between donor and acceptor groups. For DR1 and DR73 molecules, $\alpha_{x x}$ component of polarizability dominates in contrast to azobenzene where $\alpha_{z z}$ dominates. The HOMO $\rightarrow$ LUMO transition during excitation contributes to the peak molecular response in simulated UV-Visible spectra. The high polarizability response of selected D- $\pi$-A conjugated molecules in comparison to parent molecule suggests that these molecules are promising candidates for tailor-made photonic and optoelectronic device development.
\end{abstract}

Key Words: DFT, TD-DFT, Optical properties, UV-Visible Spectra, D- $\pi$-A organic dye molecules, Disperse Red 1, Disperse Red 73

\section{Introduction}

Novel organic optical materials have garnered significant scientific interest due to their potential applications in optical data storage [1], molecular photovoltaics [2,3], laser technology [4], organic photonics [5], and nonlinear optics [6]. It has been observed that organic molecules exhibit large intrinsic optical responses and are therefore promising candidates for optoelectronic and photonic applications $[7,8]$. Organic molecules are increasingly replacing inorganic optical materials due to their higher photoelectric coefficients, lower dielectric constants, low production cost, and flexibility toward design and fabrication [9]. Among organic molecules, D- $\pi$-A conjugated organic dye molecules have received considerable attention due to their unique optical properties and has been shown to be suitable candidates for sensitizer in dye-sensitized solar cells (DSSC) applications [10,11].

For designing practical optical devices, D- $\pi$-A conjugated organic dye molecules are incorporated in polymeric matrices and have been observed to show high optical response properties. Nahata et al. [12] studied the non-linear optical properties of Disperse Red 1 (DR1) covalently functionalized to a methyl methacrylate (PMMA) backbone polymer. DR1-functionalized PMMA copolymer undergoes reversible Simple Harmonic Generation (SHG) switching due to molecular-level changes in the pendant DR1 units in the film [13]. Polymer composite poly(vinyl carbazole):trinitrofluorenone/DR1 has been shown to possess dual grating formation due to simultaneous photorefractivity and photoisomerization [14]. The substantial third-order non-linear effect is experimentally observed for DR1 dye covalently doped organic-inorganic hybrid films [15]. Thus, polymers functionalized with disperse red molecules can be used to control the electrical and optical properties at the molecular levels and thereby help develop

kriti.ipu@gmail.com (K.Batra*Corresponding author) 
new technologies for high data storage applications [16].

Quantum chemical methods act as an excellent computational tool to predict the optical properties of these organic molecules and identify the structure-property relationship within these molecules [17]. Some computational research studies have been previously performed for DR1 molecule. The molecular structure and absorption spectrum of the DR1 dye molecule has been computationally studied by Ojanen et al. [18]. Poprawa-Smoluch [19] investigated the photoisomerization property of DR1 with the help of Transient Absorption Spectroscopy and quantum chemical calculations.

Recent molecular modelling studies of organic molecules illustrate their unique optical properties and confirm them to be suitable candidates for nanophotonics, terahertz photonics and optical image processing [20-23]. In this study, we investigate the molecular response of D- $\pi$-A conjugated organic dye molecules (Disperse Red 1 (DR1) and Disperse Red 73 (DR73)) using Time-Dependent Density Functional Theory (TD-DFT) and compare it with parent azobenzene structure to highlight the effect of substituents. For accurate prediction of response properties, electron correlation within the molecular system must be taken into account. Under TD-DFT formalism, functionals are used to approximate electron correlation within the system, and hence the selection of appropriate functional is critical for accurate prediction of optical properties. Conventional functionals overestimate the optical properties for large $\pi$-conjugated systems. However, long-range corrected functionals incorporate long-range effects and therefore describe the diffuse regions of charge distribution adequately [24]. Consequently, we have considered long-range corrected functionals for estimating the structure and response of selected molecules. The predictive performance of functionals is theoretically estimated and compared with experimental data avaliable.

Our objective is to perform a systematic computational analysis on Disperse Dyes (DR1 and DR73) and Azobenzene molecules using both long-range corrected functionals (CAM-B3LYP, LC- $\omega$ PBE and $\omega \mathrm{B} 97 \mathrm{XD})$ and, for comparison purposes, the popular hybrid B3LYP functional in conjunction with 6$31 \mathrm{G}(\mathrm{d}, \mathrm{p})$ basis set $[25]$. The molecular structure, vertical excitation energy, transition dipole moments, optical properties (static and dynamic polarizability, refractive index) have been reported. We aim to assess the quality of these functionals in predicting the optical properties and the substituent effect on the optical properties of target molecules.

\section{Theoretical Background}

\subsection{Optical Properties of Molecules}

One of the most crucial aspects determining practical applications of these molecular systems is their optical properties. The optical properties reveal the interaction of the molecule with the external electric field. The energy of the molecule varies with the external electric field. For static external electric field, this shift in energy can be defined as:

$$
E=E_{0}-\mu_{i} F_{i}-\frac{1}{2} \alpha_{i j} F_{i} F_{j}
$$

where $\mu_{i}, \alpha_{i j}$ are the dipole moment and polarizability components of the molecule. $E_{0}$ is the ground state energy of the molecule, $F_{i}$ and $F_{j}$ are electric field vectors along i and j direction respectively.

For dynamic fields, the change in dipole moment $(\mu(\omega))$ as a function of frequency $\omega$ is defined as

$$
\mu(\omega)=\mu^{(0)}+\alpha_{i j}(\omega) F_{j}(\omega)
$$

The electric dipole moment $(\mu)$ is a measure of the charge density within the molecule. It also indicates the strength of intermolecular reactions, where higher the dipole moment more substantial are the intermolecular reactions. However, the dipole moment contains no information about the response of the ground state wave function to the electric field as it can be computed from the zero-field density alone. The response of a molecule to an external electric field is encoded within the molecule's polarizability and hyperpolarizability components. Polarizability determines the linear response of the molecule to the external electric field whereas first-order hyperpolarizability models the molecule's quadratic response. 
Molecular polarizability is one of the most fundamental optical properties of conjugated systems and can be calculated using Sum-Over-States (SOS) formalism. Within this method, for all single excited electronic states, their corresponding vertical excitation energy and transition dipole moment from the ground state is used to calculate the static and dynamic polarizability of the molecule as follows:

$$
\begin{gathered}
\alpha_{i j}(0)=2 \sum_{n} \frac{\mu_{i}^{g n} \mu_{j}^{n g}}{\hbar \omega_{g n}} \\
\alpha_{i j}(\omega)=\sum_{n}\left(\frac{\mu_{i}^{g n} \mu_{j}^{n g}}{\hbar \omega_{g n}-\hbar \omega}+\frac{\mu_{j}^{g n} \mu_{i}^{n g}}{\hbar \omega_{g n}+\hbar \omega}\right) \\
\alpha_{i j}(\omega)=\sum_{n}\left(\frac{\left(2 \hbar \omega_{g n}\right) \mu_{i}^{g n} \mu_{j}^{n g}}{\hbar^{2} \omega_{g n}^{2}-\hbar^{2} \omega^{2}}\right) \\
\alpha_{i j}(\omega)=2 \sum_{n} \frac{\mu_{i}^{g n} \mu_{j}^{n g}}{\hbar \omega_{g n}-\frac{\omega^{2}}{\omega_{g n}}}
\end{gathered}
$$

where $\alpha_{i j}(0)$ and $\alpha_{i j}(\omega)$ are static and dynamic polarizability respectively, $\mu_{i}^{g n}$ is the transition dipole moment from ground state to $\mathrm{n}^{t h}$ excited state in the $\mathrm{i}^{\text {th }}$ direction, $\mu_{j}^{n g}$ is the transition dipole moment from $\mathrm{n}^{t h}$ excited state to ground state in the $\mathrm{j}^{\text {th }}$ direction, $\hbar \omega_{g n}=E_{n}-E_{g}$ is the transition energy and $\hbar \omega$ is the photon energy.

The components of molecular polarizability tensor thus calculated can be utilized further to estimate the isotropic polarizability $(\alpha)$ and anisotropic polarizability $(\Delta \alpha)$ using the following equations

$$
\begin{gathered}
\alpha=\frac{1}{3}\left(\alpha_{x x}+\alpha_{y y}+\alpha_{z z}\right) \\
\Delta \alpha=\frac{1}{\sqrt{2}} \sqrt{\left(\alpha_{x x}-\alpha_{y y}\right)^{2}+\left(\alpha_{y y}-\alpha_{z z}\right)^{2}+\left(\alpha_{z z}-\alpha_{x x}\right)^{2}+6\left(\alpha_{x z}^{2}+\alpha_{x y}^{2}+\alpha_{y z}^{2}\right)}
\end{gathered}
$$

As the electromagnetic field passes through a molecular gas, it induces motion in the electrons within the molecule. The moving electrons generate a field which modifies the source field within the molecular gas. Thus, the total field experienced by an electron is due to the source field and the field of all other moving electrons within the molecule. Thus, the field within the molecular gas medium appears to be moving with different velocity than the source field. This variation in field velocity is quantifiable in terms of the refractive index of the molecular gas medium. This property is of practical importance for optical fibers, optical switching devices, and photorefractive materials. For these devices, the knowledge of the refractive index of the material is an essential parameter in the manufacturing process.

The refractive index of a molecular medium can be estimated using the calculated value of isotropic polarizability $(\alpha(\omega))$ of an isolated molecule using the Lorentz-Lorenz equation [26] as:

$$
\frac{4 \pi}{3} N \alpha(\omega)=\frac{n^{2}(\omega)-1}{n^{2}(\omega)+2}
$$

where $\mathrm{N}$ is the average number of molecules per unit volume (Avogadro Number $=\mathrm{N}_{A}$ ), $\alpha(\omega)$ is the isotropic polarizability of an isolated molecule and $n(\omega)$ is the refractive index of medium for a field of frequency $\omega$ passing through the molecular medium. The frequency-dependent isotropic polarizability directly determines the molecule's refractive index and, therefore, can be altered by substituent effect and by using different wavelengths of the field to design materials whose refractive index can be externally controlled.

Using properties calculated using TD-DFT we can also simulate the UV-Vis spectra of molecules according to the relation:

$$
\epsilon(\lambda)=\left(1.30629274 \times 10^{8}\right) \frac{f_{i}}{10^{7} / 3099.6} \cdot \exp \left[-\left(\frac{1 / \lambda-1 / \lambda_{1}}{1 / 3099.6}\right)^{2}\right]
$$

where $\epsilon(\lambda)$ is the absorbance corresponding to external field of wavelength $\lambda, f_{i}$ is the calculated oscillator strength and $\lambda_{1}$ is the excitation wavelength of the molecule. 


\subsection{DFT functionals and its effect in simulating molecular optical properties}

To model the optical properties of molecules, DFT has emerged as a popular method due to its lower computational need and improved accuracy than traditional methods like Hartree-Fock Theory. Within the DFT formalism, functionals are used to estimate the exchange-correlation energy of electrons within the molecule. A variety of functionals with different levels of approximations from the simplest to highly sophisticated have been developed and arranged in the order of Jacob's Ladder of Density Functional Approximations [27]. The computational cost increases with the level of sophistication of approximation. Local Density Approximation (LDA) is the most basic approximation that takes into account only local densities for calculating the exchange-correlation energy. The next hierarchical approximation is the Generalized Gradient Approximations (GGA), which considers both the local densities and its gradients to estimate the exchange-correlation energies. Previous studies have been performed to assess the suitability of functionals in predicting the optical properties of organic $\pi$-conjugated molecules. Champagne et al. [28] studied the accuracy of LDA and GGA in predicting the linear polarizability of p-nitroaniline (PNA) and 4-amino-4'-nitrostilbene(ANS) conjugated systems and found that the calculated values did not match the experimental results. They suggested that to estimate the polarizability of such systems, the contribution of exchange energy is much more significant than the correlation term within the exchange-correlation energy. Moreover, the exchange contribution of the LDA and GGA functionals has been reported to overestimate the molecule's optical properties. The overestimation of these properties is due to the poor asymptotic behavior of exchange-correlation functionals.

Hybrid functionals were introduced to alleviate these shortcomings by combining general gradient approximations (GGA) with Hartree-Fock(HF) exchange integral at a constant rate. B3LYP [29] is a hybrid functional and has been popular in the field of organic chemistry for calculating molecular geometry and thermochemical properties. It is composed of Becke 1988 (B88) exchange, Lee-Yang-Parr (LYP) correlation functional, Slater (S) exchange, Vosko-Wilk-Nusiar (VWN) correlation LDA functional and HF exchange integral with three parameters.

$$
E_{x c}^{B 3 L Y P}=a_{0} E_{x}^{H F}+\left(1-a_{0}\right) E_{x}^{S}+a_{x} E_{x}^{B 88}+\left(1-a_{c}\right) E_{c}^{V W N}+a_{c} E_{c}^{L Y P}
$$

where $E_{x}^{H F}$ is the Hartree-Fock exchange contribution, and $E_{x}^{S}$ is the Slater exchange contribution, $\mathrm{E}_{x}^{B 88}$ is the Becke 1988 (B88) exchange contribution, $\mathrm{E}_{c}^{V W N}$ and $\mathrm{E}_{c}^{L Y P}$ are VWN and LYP correlation energy approximations. The values of parameters $a_{0}, a_{x}$ and $a_{c}$ are $0.2,0.72$, and 0.81 respectively. However, it has been observed that it fails to predict the response of molecules to external electric fields and charge-transfer excitations within the molecule due to its inaccurate asymptotic behavior, which decays as $\frac{-0.2}{r}$ instead of exact asymptotic decay of $\frac{-1}{r}$. This necessitates improvement of asymptotic correction to exchange functional.

Range separated hybrid (RSH) functionals were first introduced by Savin et al. [30] where the interelectron coulomb operator $\frac{1}{r_{12}}$ of LDA exchange functional was split into short range (SR) and a long range (LR) contribution.

$$
\frac{1}{r_{12}}=\underbrace{\frac{1-e r f\left(\omega r_{12}\right)}{r_{12}}}_{\mathrm{SR}}+\underbrace{\frac{\operatorname{erf}\left(\omega r_{12}\right)}{r_{12}}}_{\mathrm{LR}}
$$

However, this scheme was inapplicable to conventional GGA functionals. To overcome this deficiency a 'Long-range correction scheme' was developed for GGA functionals [31]. This technique was utilized by Yanai et al. [32] to develop CAM-B3LYP functional which uses 'Coulomb-attenuating method (CAM)' approach to split the coulomb operator. Mathematically, it can be represented by:

$$
\frac{1}{r_{12}}=\underbrace{\frac{1-\left[\alpha+\beta \cdot \operatorname{erf}\left(\omega r_{12}\right)\right]}{r_{12}}}_{\mathrm{SR}}+\underbrace{\frac{\alpha+\beta \cdot \operatorname{erf}\left(\omega r_{12}\right)}{r_{12}}}_{\mathrm{LR}}
$$

where $\alpha+\beta=0.65$.

For B3LYP functional, the Hartree-Fock(HF) exchange term remains fixed (25\%) in response to variations in the interelectronic distance $\left(\mathrm{r}_{12}\right)$. However, for long-range functionals, short-range $\left(\mathrm{r}_{12} \rightarrow 0\right)$ 
and long-range $\left(\mathrm{r}_{12} \rightarrow \infty\right)$ operators of interelectronic coulomb operator $\left(\frac{1}{r_{12}}\right)$ contributes differently in response to variations in $\mathrm{r}_{12}$. In the case of CAM-B3LYP functional, short-range, and long-range operators model $19 \%$ and $65 \% \mathrm{HF}$ exchange interaction, respectively. CAM-B3LYP provides a good estimation of excitation energies as compared to GGA and hybrid functionals [33]. Other functionals included in this study, LC- $\omega$ PBE [34] and $\omega$ B97XD [35], have been developed as long-range corrected approximations to PBE and B97X functionals with $\omega=0.4 \mathrm{Bohr}^{-1}$ and $0.2 \mathrm{Bohr}^{-1}$ values respectively (ref. Eqn. (11)). Both these functionals provide 100\% long-range HF exchange (coefficient of LR term in equation (11) is 1) in comparison to CAM-B3LYP, which includes $65 \%$ long-range HF exchange (coefficient of LR term in equation (12) is $\alpha+\beta=0.65$ ). However, they simulate short-range exchange interactions differently. LC- $\omega \mathrm{PBE}$ cannot model short-range $\mathrm{HF}$ exchange interactions. On the other hand, $\omega \mathrm{B} 97 \mathrm{XD}$ models $22 \%$ of short-range $\mathrm{HF}$ exchange and includes a dispersion correction term to account for van der Waals interactions, which is absent in LC- $\omega$ PBE functional.

In our calculations, we have considered four functionals (B3LYP, CAM-B3LYP, LC- $\omega$ PBE, and $\omega$ B97XD) to approximate the exchange-correlation energy within a molecular system. The contribution of longrange $\mathrm{HF}$ exchange within each functional required to provide improved estimates of our target molecules' linear polarizability is further analyzed. To achieve this objective, we performed the sum-over-states calculation of polarizability for 100 excited states. Therefore, we have focused on the performance of functionals in predicting optical properties of targeted organic $\pi$-conjugated molecules (Azobenzene, Disperse Red 1 and Disperse Red 73). We have also explored the effect of various substituent groups in modifying the $\pi$ electron delocalization of parent molecule by studying DR1 and DR73 molecular systems. Our study aims to provide novel insights in innovating new materials with tailor-made optical properties.

\section{Computational Details}

The ground state molecular structures of azobenzene, DR1, and DR73 molecules were optimized using Density Functional Theory (DFT) along with B3LYP, CAM-B3LYP, LC- $\omega$ PBE, and $\omega$ B97XD functionals and 6-31G(d,p) basis set. Excited-state calculations for all molecules were performed using the TD-DFT method for 100 excited states. The calculated vertical excitation energies and oscillator strengths were used to calculate the static and dynamic polarizability of target molecules using all functionals under Sum-Over-States formalism. The calculated values of dynamic polarizability were further employed to assess the refractive index of target molecules. All calculations were performed using Gaussian 16 Software package [36].

\section{Results and Discussion}

\subsection{Molecular Structure}

All molecules considered in this study are $\pi$-conjugated systems. Azobenzene $\left(\mathrm{C}_{12} \mathrm{H}_{10} \mathrm{~N}_{2}\right)$ molecule has electron distribution confined within the xy plane. In contrast, Disperse Red $1\left(\mathrm{C}_{16} \mathrm{H}_{18} \mathrm{~N}_{4} \mathrm{O}_{3}\right)$ and Disperse Red $73\left(\mathrm{C}_{18} \mathrm{H}_{16} \mathrm{~N}_{6} \mathrm{O}_{2}\right)$ molecules have substituents attached to their parent azobenzene backbone which introduces electron density distribution along the z-axis. The structures of these molecules are illustrated in Figure 1.

DR1 and DR73 molecules consist of a donor and an acceptor group attached to the azobenzene molecule. Both molecules have the same acceptor group $-\mathrm{NO}_{2}$ attached to the azobenzene parent structure. On the other hand, they differ in the donor group attached to each molecule. DR1 has $-\mathrm{N}\left(\mathrm{C}_{2} \mathrm{H}_{5}\right) \mathrm{CH}_{2} \mathrm{CH}_{2} \mathrm{OH}$ donor group whereas DR73 has $-\mathrm{N}\left(\mathrm{C}_{2} \mathrm{H}_{5}\right) \mathrm{CH}_{2} \mathrm{CH}_{2} \mathrm{CN}$ group instead (Ref. Figure 1). The presence of these substituent groups modifies the structure of the parent azobenzene molecule. The calculated optimized structures for Azobenzene and DR73 molecules, using all four functionals are compared with the experimental bond parameters of the molecules [37] [38] (Ref. Table 1).

For all molecules, the bond lengths and bond angles are broadly classified into four groups: C-C bonds, $\mathrm{C}-\mathrm{N}$ bonds, $\mathrm{N}=\mathrm{N}$ azo bond, and central $\mathrm{C}-\mathrm{C}-\mathrm{N}$ bond angles connecting the two benzene rings of azobenzene mainframe structure. Table $1(\mathrm{a})$ and 1 (b) lists the optimized bond lengths and bond angles, respec- 
tively, of these molecules calculated using B3LYP, CAM-B3LYP, LC- $\omega$ PBE, and $\omega$ B97XD functionals and $6-31 \mathrm{G}(\mathrm{d}, \mathrm{p})$ basis set. The order of calculated $\mathrm{C}-\mathrm{C}$ and $\mathrm{N}=\mathrm{N}$ bond lengths of azobenzene mainframe of the molecule follows the trend: B3LYP $>\omega \mathrm{B} 97 \mathrm{XD}>\mathrm{CAM}-\mathrm{B} 3 \mathrm{LYP}>\mathrm{LC}-\omega \mathrm{PBE}$ for all molecules considered in this study. There are two C-N bonds (N1-C4 and N2-C7) present in the Azobenzene molecule and four C-N bonds (N1-C4, N2-C7, N3-C1, and N4-C10) present in DR1 and DR73 molecules. The N1-C4 and N2-C7 bond lengths for all molecules increase in the order LC- $\omega$ PBE $>\omega B$ 97XD $>$ CAMB3LYP $>$ B3LYP. These bonds are equal in the case of the azobenzene molecule, thus making the structure symmetric. However, this bond symmetry is broken due to attachment of substituents in DR1 and DR73 with N2-C7 bond lengths longer than N1-C4 bond lengths. For azobenzene molecule, LC- $\omega$ PBE gave the closest result to experimental $\mathrm{C}-\mathrm{N}$ bond length. Furthermore, we observe that all functionals predicted azobenzene molecule's planar structure, exemplified by the $\mathrm{C} 4-\mathrm{N} 1=\mathrm{N} 2-\mathrm{C} 7$ dihedral angle reported to be $180^{\circ}$. The calculated bond angle C3-C4-N1 matched with $\mathrm{C} 12-\mathrm{C} 7-\mathrm{N} 4$ and $\mathrm{C} 5-\mathrm{C} 4-\mathrm{N} 1$ angle is equal to C8-C7-N2, indicating symmetry in bond angles of azobenzene molecule. The donor and acceptor groups in DR1 and DR73 introduces an asymmetry in electronic distribution; therefore, these angles are no longer equivalent in magnitude.

For these bond lengths and bond angles, we calculate the absolute average error $\left(\mid \sum_{n}\right.$ (experimental bond length-calculated bond length)/no. of bonds(n) |) and report the calculated values in Table 1(c). The error is not reported for the DR1 molecule due to the nonavailability of experimental results. From the table, it is evident that B3LYP predicts the least absolute average error for ground state optimized structure for all molecules. Among the long-range functionals, CAM-B3LYP predicted the molecular structure more efficiently than $\omega \mathrm{B} 97 \mathrm{XD}$ and LC- $\omega \mathrm{PBE}$ functionals.

\subsection{Excitation Energies and Optical Properties}

For any unperturbed molecular system, the ground and excited electronic states can be approximated using TD-DFT formalism. In this work, we have approximated the electronic structure using four exchange-correlation functionals: B3LYP, CAM-B3LYP, $\omega$ B97XD, and LC- $\omega$ PBE for three molecular systems (azobenzene, DR1 and DR 73) to evaluate the ground and excited states for each molecule. In the presence of an external field, the molecule is in a perturbed ground state, which can be expressed as a combination of unperturbed ground and excited states of the molecule and exhibits different electron density distribution than the unperturbed ground state. When the molecule interacts with the external field, instantaneous transitions occur from the unperturbed ground state to the perturbed ground state. This response of the molecule to the external electric field can be modelled using perturbation theory, which ultimately describes this behavior quantitatively in terms of sum-over-states polarizability tensor (Ref. equations 3-6).

The sum over states calculation requires the number of states (n) to be specified to calculate the polarizability tensor components of a molecule. For this purpose, we calculated the isotropic $(\alpha(\omega))$ and anisotropic $(\Delta \alpha(\omega))$ polarizability of azobenzene molecule using B3LYP functional and 6-31G(d,p) basis set for $\mathrm{n}=10,20,30,50,100,150,200,250$ excited states. The results are plotted in Figure 2. From the figure, we infer that the calculated values of $\alpha(\omega)$ and $\Delta \alpha(\omega)$ remain almost constant beyond $\mathrm{n}=100$. This indicates that all the important transitions responsible for the linear polarizability response of the molecule lie within 0-100 states. The transitions occurring to states above $n=100$ do not contribute appreciably to our calculations. Therefore, to maintain a balance between the computational cost of our calculations and the accuracy of our calculations we have calculated polarizability response of our target molecules by sum-over-states calculations for $\mathrm{n}=100$ excited states.

The polarizability tensor components, as defined in equation (4), depends on two factors: transition dipole moment between the ground and excited states of the molecule and the excitation energy of corresponding transitions (i.e., the energy gap between the ground and excited states). The polarizability tensor components calculated using the sum-over-state method can be further utilized to estimate the isotropic $(\alpha(\omega))$ and anisotropic $(\Delta \alpha(\omega))$ polarizability of the molecules using equations (7) and (8). Thus, the behaviour of $\alpha(\omega)$ and $\Delta \alpha(\omega)$ response functions is determined by the polarizability components $\alpha_{x x}(\omega), \alpha_{y y}(\omega)$ and $\alpha_{z z}(\omega)$.

Polarizability represents the linear response of electrons in a molecule to an external oscillating elec- 
tric field. The nature of this response is determined by the transitions occurring within the molecular system. When an external field perturbs the molecule, the response of the system is substantial when during the transition, the energy of perturbing external field $(\hbar \omega)$ matches closely with the transition energy $\left(\hbar \omega_{g n}\right)$, (Ref. equation 5) electronic transition occurs and the molecule exhibits a significant polarizability response. During this electronic transition within the molecule, transition dipole moment provides a measure of the extent of the oscillating movement of electrons within the molecule resulting from interaction with the oscillating field. If the extent of interaction is large, then from equation (4), we observe that the substantial value of TDM will result in significant polarizability tensor component indicating coupling between the molecule and external field. The TDM values of all 100 excited states for all molecules is depicted in Figure 3.

Figure 4 shows the polarizability curves of tensor components $\alpha_{x x}(\omega), \alpha_{y y}(\omega)$ and $\alpha_{z z}(\omega)$ as well as the net isotropic $(\alpha(\omega))$ and anisotropic $(\Delta \alpha(\omega))$ polarizability variation of azobenzene molecule as a function of frequency of external field $(\omega)$. These curves demonstrate the effect of functional in modelling the response of azobenzene to external electric field. In the case of azobenzene molecule, the contribution from $\alpha_{z z}(\omega)$ component is negligible when compared with the contribution from $\alpha_{x x}(\omega)$ and $\alpha_{y y}(\omega)$. Among all the polarizability components $\alpha_{y y}(\omega)$ has the largest polarizability value and therefore plays a major role in determining the response of $\alpha(\omega)$ and $\Delta \alpha(\omega)$ with respect to the external field. All functionals predict peak polarizability in $\alpha_{y y}(\omega)$ response curve at $\omega=0.152$ a.u.. The transition between ground $(|0\rangle)$ to second $(|2\rangle)$ excited state of the molecule for all functionals has the maximum value of transition dipole moment (TDM) among all transitions occurring within the molecule (Figure 3(a)), where $\left|\left\langle\mu_{y}\right\rangle\right|=2.8896$ for B3LYP, 2.8521 for CAM-B3LYP, 2.8505 for $\omega$ B97XD and 2.7614 for LC- $\omega \mathrm{PBE}$ functionals. The excitation frequency corresponding to $|0\rangle \rightarrow|2\rangle$ transition is calculated to be 0.1385 a.u. for B3LYP, 0.150 a.u. for CAM-B3LYP, 0.1504 a.u. for $\omega$ B97XD and 0.1606 a.u. for LC- $\omega$ PBE functionals. The excitation energy $\left(\omega_{02}\right)$ of $|0\rangle \rightarrow|2\rangle$ transition match closely with frequency corresponding to peak polarizability frequency $(\omega)$. Thus, $|0\rangle \rightarrow|2\rangle$ transition plays an important role in determining sum-over-state polarizability of $\alpha_{y y}(\omega)$ component for azobenzene molecule. We observe that as the percentage of long-range HF exchange increases from 25\% in B3LYP to $65 \%$ in CAM-B3LYP and subsequently to $100 \%$ in $\omega \mathrm{B} 97 \mathrm{XD}$ and LC-wPBE functionals, the predicted excitation frequency increases and TDM values decreases for $|0\rangle \rightarrow|2\rangle$ transition. In the case of $\omega \mathrm{B} 97 \mathrm{XD}$ and LC- $\omega \mathrm{PBE}$ functionals, although both have the same \% of long-range HF exchange, the predicted values of excitation energy and TDM differs. This difference in values might be due to the presence of short-range HF exchange included in $\omega \mathrm{B} 97 \mathrm{XD}$, which is absent in LC- $\omega \mathrm{PBE}$ functional.

For $\alpha_{y y}(\omega)$ component of polarizability, we observe that B3LYP, CAM-B3LYP and $\omega$ B97XD predicts negative values whereas LC- $\omega \mathrm{PBE}$ predicts positive values. The variation of $\alpha_{x x}(\omega)$ component of polarizability varies along both the positive and negative values for B3LYP, CAM-B3LYP and $\omega$ B97XD functional while remains positive for all frequencies for LC- $\omega \mathrm{PBE}$. The positive values $\alpha_{y y}(\omega)$ and $\alpha_{x x}(\omega)$ indicate that the change in dipole moment in response to oscillating electric field is along the direction of the applied electric field. However, the negative values of $\alpha_{y y}(\omega)$ and $\alpha_{x x}(\omega)$ signify change in dipole moment opposite in response to the external electric field.

Figure 5 describes the response of molecular polarizability tensors $\left(\alpha_{x x}(\omega), \alpha_{y y}(\omega)\right.$ and $\left.\alpha_{z z}(\omega)\right)$ and isotropic $(\alpha(\omega))$ and anisotropic $(\Delta \alpha(\omega))$ polarizability tensor of Disperse Red 1 molecule as a function of frequency of external electric field. From the graph, it is evident that the response of DR1 predicted by CAM-B3LYP functional for $\alpha_{x x}(\omega)$ is larger as compared to the response predicted by B3LYP, $\omega \mathrm{B} 97 \mathrm{XD}$ and LC- $\omega \mathrm{PBE}$ functionals. For $\alpha_{x x}(\omega)$ vs $\omega$ curve, two positive peaks are observed at external field frequencies $\omega=0.09$ a.u. and 0.15 a.u. for B3LYP functional. In the case of this functional, the $|0\rangle \rightarrow|2\rangle$ and $|0\rangle \rightarrow|9\rangle$ transitions occurs at excitation frequency 0.0967 a.u. and 0.15196 a.u.. Thus, the one positive peak in the curve of $\alpha_{x x}(\omega)$ vs $\omega$ at frequency 0.09 a.u. is due $|0\rangle \rightarrow|2\rangle$ transition with corresponding TDM $\left|\left\langle\mu_{x}\right\rangle\right|=3.7996$. The other positive peak in the curve at frequency 0.151 a.u. is due to $|0\rangle \rightarrow|9\rangle$ transition occuring within the molecular system with TDM $\left|\left\langle\mu_{x}\right\rangle\right|=1.0393$. The calculated plots of $\alpha_{x x}(\omega)$ using CAM-B3LYP, $\omega$ B97XD and LC- $\omega$ PBE functionals show peak polarizability at 0.114 a.u.. For these functionals, $|0\rangle \rightarrow|2\rangle$ transition requires excitation frequency of 0.1151 a.u., 0.1261 a.u. and 0.1174 a.u. respectively. Thus, in contrast with B3LYP functional which predicts two positive peaks corresponding to transitions $|0\rangle \rightarrow|2\rangle$ and $|0\rangle \rightarrow|9\rangle$, other functionals exhibits peak polarizability only due to $|0\rangle \rightarrow|2\rangle$ transition for $\alpha_{x x}(\omega)$. 
From the curves of polarizability tensor components in Figure 5, it is evident that significant contribution to $\alpha(\omega)$ and $\Delta \alpha(\omega)$ is due to $\alpha_{x x}(\omega)$, while $\alpha_{z z}(\omega)$ contributes the least. The peaks for $\alpha_{x x}(\omega)$ are confined within the frequency 0.09 - 0.2 a.u., while for $\alpha_{y y}(\omega)$ component, response extends within the range of $0.1-0.45$ a.u. This variation of each polarizability tensor components $\left(\alpha_{x x}(\omega), \alpha_{y y}(\omega)\right.$ and $\left.\alpha_{z z}(\omega)\right)$ ultimately affects the isotropic $(\alpha(\omega))$ and anisotropic $(\Delta \alpha(\omega))$ response of Disperse Red 1 molecule. The polarizability response of $\alpha(\omega)$ closely mimics the response of $\alpha_{x x}(\omega)$ due to the dominant contribution of this tensor component. The maximum peak polarizability is computed by CAM-B3LYP functional, and the least peak polarizability for $\alpha(\omega)$ and $\Delta \alpha(\omega)$ is calculated for LC- $\omega$ PBE functional. Similar to the case of azobenzene molecule, in the case of DR1, the major contributing transition is $|0\rangle \rightarrow|2\rangle$ transition (Figure 3(b)). Moreover, for both molecules, LC- $\omega$ PBE functional predicts consistently positive values for $\alpha_{x x}(\omega), \alpha(\omega)$, and $\Delta \alpha(\omega)$

Figure 6 represents variation in polarizability of Disperse Red 73 molecule in response to an external electric field of frequency $\omega$. The contribution of polarizability tensor components in the estimation of $\alpha(\omega)$ and $\Delta \alpha(\omega)$ increases as $\alpha_{x x}(\omega)>\alpha_{y y}(\omega)>\alpha_{z z}(\omega)$. The major contributing polarizibility tensor component $\alpha_{x x}(\omega)$ has maximum positive peak responses at external field frequencies $\omega=0.0911$ a.u. and 0.1518 a.u. for B3LYP and $\omega=0.1139$ for CAM-B3LYP, wB97XD, and LC-wPBE. In the case of B3LYP functional, the excitation frequencies for DR73 are calculated to be $\omega_{02}=0.097$ a.u. and $\omega_{09}=$ 0.1517 a.u.. These values of excitation frequencies are close to the frequencies of positive peak sum over state $\alpha_{x x}(\omega)$ polarizability (Ref equation 4) observed for B3LYP functional. Thus, in the case of B3LYP functional, instantaneous transitions $|0\rangle \rightarrow|2\rangle$ and $|0\rangle \rightarrow|9\rangle$ gives rise to positive peak polarizability of DR73 molecule. For functionals, with increased \% of long range HF exchange, positive peak polarizability is observed at frequency $\omega=0.1139$ a.u.. The excitation frequency of $|0\rangle \rightarrow|2\rangle$ transition is estimated using TD-DFT to be 0.114 a.u. for CAM-B3LYP, 0.116 a.u. for $\omega$ B97XD and 0.1245 a.u. for LC-wPBE. The corresponding TDM $\left|\left\langle\mu_{x}\right\rangle\right|$ of this transition (listed in Table 2) is maximum of all calculated TDM between ground and excited states along the x-axis. The effect of $\alpha_{x x}(\omega)$ polarizability component can be directly observed at the peak $\alpha(\omega)$ and $\Delta \alpha(\omega)$ where for CAM-B3LYP, $\omega$ B97XD and LC- $\omega$ PBE functionals, peak polarizability is observed at $\omega=0.1139$ a.u.. In the case of B3LYP, peak polarizability for $\alpha(\omega)$ and $\Delta \alpha(\omega)$ are observed at $\omega=0.0911$ a.u. and 0.1518 a.u., close to calculated excitation frequencies. Thus, similar to DR1 molecule, in the case of DR73 it is the $|0\rangle \rightarrow|2\rangle$ transition is crucial in determining the maximum polarizability response of these molecules (Figure 3(c)).

Two major transitions occur in the case of azobenzene and substituted azobenzene molecules, termed as $\pi \rightarrow \pi^{*}$ and $\mathrm{n} \rightarrow \pi^{*}$. In the trans configuration of the molecule (the one we have considered in our study), $\pi \rightarrow \pi^{*}$ is the dominant transition occurring at $325 \mathrm{~nm}$ and $\mathrm{n} \rightarrow \pi^{*}$ is a much weaker transition occurring at 450nm [39]. In the case of DR1 and DR73 molecules, similar $\pi \rightarrow \pi^{*}$ dominant transitions take place at wavelength $406 \mathrm{~nm}$ [40] and $521 \mathrm{~nm}$ [41] respectively. We compare this experimentally observed dominant transition $\pi \rightarrow \pi^{*}$ in azobenzene and DR1 with the transition $|0\rangle \rightarrow|2\rangle$ observed in our calculated results of azobenzene and DR1. The analysis of sum-over-states polarizability curves for azobenzene, DR1, and DR73 molecules reveal that it is $|0\rangle \rightarrow|2\rangle$ transition, which contribute to maximum polarizability of molecules. The calculated excitation wavelength of $|0\rangle \rightarrow|2\rangle$ transition for the target molecules is closest to the experimental $\pi \rightarrow \pi *$ transition excitation wavelengths, and both excitation wavelengths (theoretically calculated and experimental) are listed in Table 2.

In the case of $\mathrm{D}-\pi$-A molecules, intramolecular charge transfer occurs from the donor to the acceptor group, which is mediated by the central azobenzene backbone. The donor group feeds the electron contribution to the azobenzene mainframe, which is further withdrawn by the acceptor group. Thus $\mathrm{D}-\pi$-A system has charge distribution over an extended outer region in comparison to the Azobenzene parent molecule. The electrons residing in the outer regions (donor and acceptor electrons) interact with the exchange hole, which is situated at a large distance from the electron. This electrostatic interaction of electrons with its holes in the donor and acceptor groups separated by substantial distance can be modelled only by the inclusion of non-local HF interaction. Therefore, long-range functionals yield better performance in modelling the molecular response of $\mathrm{D}-\pi$-A molecules.

In the presence of external field, the variation in TDM for azobenzene molecule is observed along the xy plane for all functionals i.e., $\left\langle\mu_{z}\right\rangle=0$. However, substituted azobenzene molecules (DR1 and DR73) 
have donor and acceptor groups attached to the parent azobenzene backbone. These substituent groups introduce electron density distribution in the z plane, which results in $\left\langle\mu_{z}\right\rangle \neq 0$ (Ref. Table 2). Therefore, substituent group attachment to the azobenzene molecule introduces a slight variation of dipole moment along the z-axis, thereby increasing the net TDM value of substituted molecules compared to the parent molecule. Moreover, for DR1 and DR73, the substituent groups decrease the excitation energy of the molecule. Changes in excitation energy and TDM directly affect the static and dynamic polarizability of these molecules.

The static polarizability of target molecules has been reported in Table 3. Analysis with different functionals reveals that B3LYP consistently predicts a large value of static polarizability, whereas LC$\omega \mathrm{PBE}$ predicts the least values. The static polarizability predicted using CAM-B3LYP, and $\omega \mathrm{B} 97 \mathrm{XD}$ functional are numerically larger than LC- $\omega \mathrm{PBE}$ but are in close approximation to each other. This behavior is observed for all studied molecules. It indicates that an increase in long-range HF exchange from $65 \%$ to $100 \%$ resulted in slight decreased value of linear static response of the molecule. We also observe that both $\omega \mathrm{B} 97 \mathrm{XD}$ and LC- $\omega \mathrm{PBE}$ functionals have $100 \%$ long-range HF exchange functional. However, LC- $\omega \mathrm{PBE}$ values are lower than $\omega \mathrm{B} 97 \mathrm{XD}$ values. This behavior may be observed in molecules due to the presence of $\%$ short-range HF exchange in $\omega$ B97XD which is lacking in LC- $\omega$ PBE functional.

Figure 7 shows the variation of the refractive index of molecules (calculated using equation 9) as a function of frequency $\omega$ of the external field. For azobenzene, DR1 and DR73, $\omega$ B97XD predicts substantial refractive index values at $\omega=0.1139$ a.u.. in comparison to other functionals and thus might not be suitable to predict refractive index response within the theoretical framework considered in our study (equations 1-9). The refractive index is dependent on the isotropic polarizability $\alpha(\omega)$ of the molecule whose response is governed by $\alpha_{y y}(\omega)$ in case of azobenzene molecule and $\alpha_{x x}(\omega)$ in case of DR1 and DR73. These polarizability responses are due to molecular electronic transitions within the molecule among which $|0\rangle \rightarrow|2\rangle$ transition contributes significantly. Each functional simulates this molecule transition uniquely and thus predicts different responses of polarizability and refractive index of the molecule. Therefore, choosing an adequate functional is necessary to study the response of $\pi$-conjugated molecular systems of practical importance.

\subsection{UV- Visible Spectra}

Figures 8, 9, and 10 represent the UV-Visible spectra of Azobenzene, Disperse Red 1, and Disperse Red 73 molecules. The maximum peak excitation wavelength calculated for all molecules corresponds to $|0\rangle \rightarrow|2\rangle$. A general trend is observed in the UV Visible spectra of all three molecules. As the $\%$ of long-range HF exchange increases from $25 \%$ in B3LYP to $65 \%$ in CAMB3LYP and $100 \%$ in $\omega$ B97XD and LC- $\omega \mathrm{PBE}$, the peak excitation wavelength for all molecules decreases. Although both $\omega \mathrm{B} 97 \mathrm{XD}$ and LC- $\omega$ PBE have the same percentage of long-range HF exchange, we observe a shift in maximum excitation wavelength of the molecules due to the short-range range HF exchange-correlation which is absent in the case of LC- $\omega$ PBE. This behavior clearly illustrates that HF exchange interaction plays a crucial role in modeling molecules' response.

For all molecules, B3LYP functional, we observe two dominant peak excitation wavelengths. For the azobenzene molecule, these peaks are observed at $224.80 \mathrm{~nm}$ and $328.93 \mathrm{~nm}$, i.e., the peaks are observed in the UV range $(200-400 \mathrm{~nm})$ of the spectrum. In the case of substituted molecules DR1 and DR73, B3LYP predicts maximum excitation wavelengths to be $248.80 \mathrm{~nm}$ and $471.04 \mathrm{~nm}$ for DR1; $346.45 \mathrm{~nm}$ and $470.81 \mathrm{~nm}$ for DR73 molecule. Therefore, in the case of B3LYP functional, due to the presence of substituents, the excitation peaks shifted towards the visible end of the spectra.

A decrease in maximum excitation wavelength in the UV-Visible spectra of the molecules calculated using $\omega$ B97XD when compared with CAM-B3LYP. For azobenzene molecule, CAM-B3LYP and $\omega$ B97XD predict maximum excitation wavelength at $303.77 \mathrm{~nm}$ and $302.79 \mathrm{~nm}$, respectively. This peak shifts to $395.90 \mathrm{~nm}$ and $387.91 \mathrm{~nm}$ for DR1 molecule; $399.65 \mathrm{~nm}$ and $365.96 \mathrm{~nm}$ for DR73 molecule, i.e., addition of donor and acceptor substituents increases the maximum excitation wavelength. However, all excitations lie with the UV region of the spectra. LC- $\omega$ PBE predicts the lowest excitation wavelength at $383.66 \mathrm{~nm}, 361.33 \mathrm{~nm}$, and $391.83 \mathrm{~nm}$ for Azobenzene, Disperse Red 1, and Disperse Red 73 molecules, respectively. Unlike other functionals, here DR1 shows decrease in excitation wavelength 
while maximum excitation wavelength increases for DR73 molecule.

These excitations are observed due to orbital transitions ocurring within the molecule. For all peak excitations of all molecules we report the contributing orbital transitions and the corresponding oscillator strengths in Table 4. For all molecules long-range functionals predicts same orbital transitions $(\mathrm{H}(\mathrm{HOMO}) \rightarrow \mathrm{L}(\mathrm{LUMO})$ in contrast to B3LYP functional. In the case of these functionals, the percentage contribution of the transition $\mathrm{H} \rightarrow \mathrm{L}$ decreases with increase in the $\%$ of long-range HF exchange. For B3LYP functional we observe two dominant peak excitation wavelengths. For the excitation wavelength corresponding to maximum absorbance $(\epsilon)$, the percentage contribution of the transition $\mathrm{H}(\mathrm{HOMO}) \rightarrow$ $\mathrm{L}(\mathrm{LUMO})$ is still the dominant orbital contribution to the electron excitation.

To verify the validity of our experimental results, we compared the calculated excitation wavelength with available experimental wavalengths reported in Table 2. The correlation plot comparing the values is shown in Figure 11 where we report the correlation factor $\left(\mathrm{R}^{2}\right)$ and Root Mean Square Error (RMSE) parameters of the best fit for all functionals. It is clearly evident from the graph that long-range functionals report better $\mathrm{R}^{2}$ values in comparison to B3LYP functionals. Moreover, among the long-range functional, CAM-B3LYP results exhibit the least error and therefore is better suited for modelling molecular excitations of azobenzene and its substituted molecules.

The superior performance of long-range functionals can be understood in terms of orbital transitions occurring within the molecule. The maximum contributing orbital transition $\mathrm{H} \rightarrow \mathrm{L}$ (or $\pi \rightarrow \pi^{*}$ transition) is accompanied by charge transfer upon excitation over long-range and can therefore be modelled only by the inclusion of appropriate balance of short-range and long-range HF exchange in the functionals. Among the three long-range functionals considered in this study, CAM-B3LYP gave the best results.

\section{Conclusion}

In this study, we used DFT to study molecular structure and TD-DFT to analyze the excitation energies, transition dipole moments, molecular polarizability (static and dynamic), and refractive index of azobenzene and its substituted molecules (DR1 and DR73). The analysis was made using hybrid B3LYP functional as well as long-range separated DFT functionals (CAM-B3LYP, $\omega$ B97XD, and LC- $\omega$ PBE). The long-range separated functionals had a different percentage of long-range HF exchange character associated with each functional. B3LYP and CAM-B3LYP functionals predict the molecular structure with the least average error for all molecules. In the case of the azobenzene molecule, the maximum change in polarizability is due to $\alpha_{y y}(\omega)$ component of polarizability tensor, whereas for substituted molecules DR1 and DR73, $\alpha_{x x}(\omega)$ contribute to maximum polarizability response. It can be concluded that for azobenzene, DR1 and DR73, $|0\rangle \rightarrow|2\rangle$ transition is the dominant transition contributing to dynamic polarizability response of these $\pi$-conjugated molecule to an external field. From our analysis, we also report that for modelling $|0\rangle \rightarrow|2\rangle$ excitation in the UV-Visible spectra, long-range functionals outperformed B3LYP functional. Among the long-range functional, CAM-B3LYP gave the closest result to the experimental value with the least RMSE value. The performance of CAM-B3LYP indicates its ability to model the charge transfer processes in substituted molecules upon excitation. This inclusion of long-range HF exchange energy is also crucial for accurate prediction of polarizability response where $|0\rangle \rightarrow|2\rangle$ transition contributed to the dominant response. We also observed that introducing substitution within the parent molecule can be used to control the molecular polarizability and tune it for practical applications.

The $\pi$-electron conjugation of $\mathrm{D}-\pi$-A conjugated organic dye molecules contributes to the remarkable performance of these molecules for optoelectronic applications. Our study demonstrates that selecting the right theoretical model is essential for predicting optical properties of organic conjugated molecules. Moreover, this study also sheds light on tailoring the optical properties by attaching donor and acceptor groups to azobenzene based molecules. These studies are essential for studying the interaction of organic conjugated molecules with light. These light-molecule interactions are at the heart of many novel technologies like optoelectronic logic circuits, piezoelectric materials, organic molecular circuits, optical storage devices, and noninvasive imaging techniques. 


\section{Acknowledgments}

Dr. Kriti Batra is thankful to Guru Gobind Singh Indraprastha University for research grant under the Faculty Research Grant Scheme (FRGS) for the year 2019-20 (F.No. GGSIPU/DRC/FRGS/2019/1553/12). Garima Chanana is grateful to Guru Gobind Singh Indraprastha University for providing financial support under Short Term Research Fellowship (STRF) scheme (L.No GGSIPU/DRC/Ph.D./2018/1288)

\section{Declarations}

\section{Funding}

This study was financially supported by Guru Gobind Singh Indraprastha University.

\section{Conflict of Interest}

The authors declare that they have no conflict of interest.

\section{Ethics approval}

This article does not contain any studies with human participants or animals performed by any of the authors.

\section{Consent to Participate}

For this type of study, no consent is required as there is no humans or animal participation in the study.

\section{Consent for Publication}

For this type of study, no consent is required as there is no humans or animal participation in the study.

\section{Availability of Data and Material}

All data analyzed during this study are reported within this article.

\section{Code Availability}

All calculations have been performed using the licensed Gaussian 16 Software Application.

\section{Authors' Contributions}

Dr. Kriti Batra (Corresponding Author)- Conceptualization of idea of study, Software-implementation of computer code, Formal Analysis, Provision of Resources, Supervision, Review and editing the draft, Project administration and Funding acquisition.

Garima Chanana (First author)- Validation of results, Investigation-performing calculations, Data curation-maintaining research data for initial and final use, writing original draft preparation, Visualizationpresentation of results.

\section{References}

[1] Mustroph H, Stollenwerk M, Bressau V (2006) Current developments in optical data storage with organic dyes. Angew. Chem. Int. Ed. 45:2016-2035. https://doi.org/10.1002/anie.200502820

[2] Mishra A, Fischer MK, Bäuerle P (2009) Metal-free organic dyes for dye-sensitized solar cells: From structure: Property relationships to design rules. Angew. Chem. Int. Ed. 48:2474-2499. https://doi.org/10.1002/anie.200804709 
[3] Kakiage K, Aoyama Y, Yano T, Otsuka T,Kyomen T, Unno M, Hanaya M (2014) An achievement of over 12 percent efficiency in an organic dye-sensitized solar cell. ChemComm. 50:6379-6381. https://doi.org/10.1039/C4CC02192D

[4] Kuehne AJ, Gather MC (2016) Organic lasers: recent developments on materials, device geometries, and fabrication techniques. Chem. Rev. 116:12823-12864. https://doi.org/10.1021/acs.chemrev.6b00172

[5] Clark J, Lanzani G (2010) Organic photonics for communications. Nat. Photonics 4:438-446 https://doi.org/10.1038/nphoton.2010.160

[6] Haley JE (2020) Development of Organic Nonlinear Optical Materials for Light Manipulation. In Women in Aerospace Materials, Springer, Cham. 35-47. https://doi.org/10.1007/978-3-030-407797_3

[7] Pramodini S, Poornesh P (2014) Third-order nonlinear optical response of indigo carmine under $633 \mathrm{~nm}$ excitation for nonlinear optical applications. Opt. Laser Technol. 63:114-119. https://doi.org/10.1016/j.optlastec.2014.04.007

[8] Kim TD, Lee KS (2015) D- $\pi$-A Conjugated Molecules for Optoelectronic Applications. Macromol. Rapid Commun. 36:943-958. https://doi.org/10.1002/marc.201400749

[9] Khan MU, Ibrahim M, Khalid M, Braga AAC, Ahmed S, Sultan A (2019) Prediction of Second-Order Nonlinear Optical Properties of D- $\pi-\mathrm{A}$ Compounds Containing Novel Fluorene Derivatives: A Promising Route to Giant Hyperpolarizabilities. J. Clust. Sci. 30:415-430. https://doi.org/10.1007/s10876-018-01489-1

[10] Bouzineb Y, Slimi A, Raftani M, Fitri A, Benjelloun AT, Benzakour M, Mcharfi M, Bouachrine M (2020) Theoretical study of organic sensitizers based on 2, 6-diphenyl-4Hpyranylidene/1, 3, 4-oxadiazole for dye-sensitized solar cells. J. Mol. Model. 26:1-12 (2020) https://doi.org/10.1007/s00894-020-04611-1

[11] El Mzioui S, Bouzzine SM, Sidir İ, Bouachrine M, Bennani MN, Bourass M and Hamidi M (2019) Theoretical investigation on $\pi$-spacer effect of the $\mathrm{D}-\pi-\mathrm{A}$ organic dyes for dyesensitized solar cell applications: A DFT and TD-BHandH study. J. Mol. Model. 25:92. https://doi.org/10.1007/s00894-019-3963-1

[12] Nahata A, Shan J, Yardley JT, Wu C (1993) Electro-optic determination of the nonlinear-optical properties of a covalently functionalized Disperse Red 1 copolymer. J. Opt. Soc. Am. B 10:15531564. https://doi.org/10.1364/JOSAB.10.001553

[13] Coe BJ (1999) Molecular materials possessing switchable quadratic nonlinear optical properties. Chem. Eur. J. 5:2464-2471. https://doi.org/10.1002/(SICI)1521-3765(19990903)5:9/2464::AIDCHEM2464>3.0.CO;2-L

[14] Kippelen B, Peyghambarian N, Lyon SR, Padias AB, Hall HK (1994) Dual-grating formation through photorefractivity and photoisomerization in azo-dye-doped polymers. Opt. Lett. 19:68-70. https://doi.org/10.1364/OL.19.000068

[15] Li J, Jiang P, Wei C, Shi J (2008) Linear and nonlinear optical properties of covalently bound CI Disperse Red 1 chromophore/silica hybrid film. Dyes Pigm. 78:219-224. https://doi.org/10.1016/j.dyepig.2007.12.005

[16] Blasco E, Piñol M, Berges C, Sánchez-Somolinos C, Oriol L (2019) Smart polymers for optical data storage, Smart Polymers and their Applications. Woodhead Publishing 567-606. https://doi.org/10.1016/B978-0-08-102416-4.00016-8

[17] Wu J, Wang W, Wang N, He J, Deng G, Li Z, Zhang X, Xiao H, Chen K (2018) Structure-property analysis of julolidine-based nonlinear optical chromophores for the optimization of microscopic and macroscopic nonlinearity. Phys. Chem. Chem. Phys. 20:23606-23615. https://doi.org/10.1039/C8CP04470H 
[18] Ojanen J, Rantala TT (2009) Electronic structure and absorption spectrum of Disperse Red 1: comparison of computational approaches. Open Chem. Phy. J. 2:37-46. https://doi.org/10.2174/1874412500902010037

[19] Poprawa-Smoluch M, Baggerman J, Zhang H, Maas HP, De Cola L, Brouwer AM (2006) Photoisomerization of Disperse Red 1 studied with transient absorption spectroscopy and quantum chemical calculations. J. Phys. Chem. A 110:11926-11937. https://doi.org/10.1021/jp054982b

[20] Bhalekar SB, Bhagwat AA, Sekar N (2020) Orange-Red Fluorescent (Partially Rigidified) Donor- $\pi$-(rigidified)-Acceptor System-Computational Studies. J. Fluoresc. 30:565-579. https://doi.org/10.1007/s10895-020-02506-1

[21] Bhalekar S, Avhad K, Sekar N (2020) Deep Red emitting dicyanovinylene isophorone based chromophores: Combined synthesis, optical properties, viscosity sensitivity, and DFT studies. J. Photochem. Photobiol. A 391:112389. https://doi.org/10.1016/j.jphotochem.2020.112389

[22] Boukabcha N, Djafri A, Megrouss Y, Tamer Ö, Avcı D, Tuna M, Dege N, Chouaih A, Atalay Y, Hamzaoui F (2019) Synthesis, crystal structure, spectroscopic characterization and nonlinear optical properties of (Z)-N'-(2, 4-dinitrobenzylidene)-2-(quinolin-8-yloxy) acetohydrazide. J. Mol. Struct. 1194:112-123. https://doi.org/10.1016/j.molstruc.2019.05.074

[23] Arığlu Ç, Tamer Ö, Avcı D, Atalay Y (2018) Optimized geometry, spectroscopic characterization and nonlinear optical properties of carbazole picrate: a density functional theory study. Indian J. Phys. 92:1613-1621. https://doi.org/10.1007/s12648-018-1258-5

[24] Jacquemin D, Perpète EA, Medved M, Scalmani G, Frisch MJ, Kobayashi R, Adamo C (2007) First hyperpolarizability of polymethineimine with long-range corrected functionals. J. Chem. Phys. 126:191108. https://doi.org/10.1063/1.2741246

[25] Helgaker T, Jorgensen P, Olsen J (2014) Chapter 8, Gaussian Basis Sets, Molecular electronicstructure theory. John Wiley and Sons

[26] Lorentz HA (1916) The Theory of Electrons and Its Applications to the Phenomena of Light and Radiant Heat: A Course of Lectures Delivered in Columbia University, New York in March and April, 1906. Leipzig: B.G.Teubner

[27] Perdew JP, Schmidt K (2001) Jacob's ladder of density functional approximations for exchange correlation energy. AIP Conf. Proc. American Institute of Physics 577:1. https://doi.org/10.1063/1.1390175

[28] Champagne B, Perpète EA, Jacquemin D, Van Gisbergen SJ, Baerends EJ, Soubra-Ghaoui C, Robins KA, Kirtman B (2000) Assessment of conventional density functional schemes for computing the dipole moment and hyperpolarizabilities of push-pull $\pi$ conjugated systems. J. Phys. Chem. A 104:4755-4763. https://doi.org/10.1021/jp993839d

[29] Becke AD (1993) Density-functional thermochemistry. III. The role of exact exchange. J. Chem. Phys. 98:5648. https://doi.org/10.1063/1.464913

[30] Savin A (1996) Recent Developments and Applications of Modern Density Functional Theory, Ed. J. M. Seminario (Elsevier, Amsterdam) p. 327.

[31] Iikura H, Tsuneda T, Yanai T, Hirao K (2001) A long-range correction scheme for generalized-gradient-approximation exchange functionals. J. Chem. Phys. 115:3540. https://doi.org/10.1063/1.1383587

[32] Yanai T, Tew DP, Handy NC (2004) A new hybrid exchange-correlation functional using the Coulomb-attenuating method (CAM-B3LYP). Chem. Phys. Lett. 393:51-57. https://doi.org/10.1016/j.cplett.2004.06.011

[33] Peach MJ, Benfield P, Helgaker T, Tozer DJ (2008) Excitation energies in density functional theory: An evaluation and a diagnostic test. J. Chem. Phys. 128:044118. https://doi.org/10.1063/1.2831900 
[34] Vydrov OA, Scuseria GE (2006) Assessment of a long-range corrected hybrid functional. J. Chem. Phys. 125:234109. https://doi.org/10.1063/1.2409292

[35] Chai JD, Head-Gordon M (2008) Long-range corrected hybrid density functionals with damped atom-atom dispersion corrections. Phys. Chem. Chem. Phys. 10:6615-6620. https://doi.org/10.1039/B810189B

[36] Gaussian 16, Revision A.03 (2016) Frisch MJ, Trucks GW, Schlegel HB, Scuseria GE, Robb MA, Cheeseman JR, Scalmani G, Barone V, Petersson GA, Nakatsuji H, Li X, Caricato M, Marenich AV, Bloino J, Janesko BG, Gomperts R, Mennucci B, Hratchian HP, Ortiz JV, Izmaylov AF, Sonnenberg JL, Williams-Young D, Ding F, Lipparini F, Egidi F, Goings J, Peng B, Petrone A, Henderson T, Ranasinghe D, Zakrzewsk VG, Gao J, Rega N, Zheng G, Liang W, Hada M, Ehara M, Toyota K, Fukuda R, Hasegawa J, Ishida M, Nakajima T, Honda Y, Kitao O, Nakai H, Vreven T, Throssell K, Montgomery JA Jr, Peralta JE, Ogliaro F, Bearpark MJ, Heyd JJ, Brothers EN, Kudin KN, Staroverov VN, Keith TA, Kobayashi R, Normand J, Raghavachari K, Rendell AP, Burant JC, Iyengar SS, Tomasi J, Cossi M, Millam JM, Klene M, Adamo C, Cammi R, Ochterski JW, Martin RL, Morokuma K, Farkas O, Foresman JB, Fox DJ, Gaussian, Inc., Wallingford CT.

[37] Bouwstra JA, Schouten A, Kroon J (1983) Structural studies of the system transazobenzene/trans-stilbene. I, A reinvestigation of the disorder in the crystal structure of trans-azobenzene $\left(\mathrm{C}_{12} \mathrm{H}_{10} \mathrm{~N}_{2}\right)$, Acta Crystallogr. C Struct. Chem. 39:1121-1123. https://doi.org/10.1107/S0108270183007611

[38] Lee JE, Kim HJ, Han MR, Lee SY, Jo WJ, Lee SS, Lee JS (2009) Crystal structures of CI Disperse red 65 and CI Disperse red 73. Dyes Pigm. 80:181-186. https://doi.org/10.1016/j.dyepig.2008.07.001

[39] Cho EN, Zhitomirsky D, Han GG, Liu Y, Grossman JC (2017) Molecularly engineered azobenzene derivatives for high energy density solid-state solar thermal fuels. ACS Appl. Mater. Interfaces 9: 8679-8687. https://doi.org/10.1021/acsami.6b15018

[40] Uliana CV, Garbellini GS, Yamanaka H (2012) Spectrophotometric evaluation of the behavior of disperse red 1 dye in aqueous media and its interaction with calf thymus ds-DNA. J. Braz. Chem. Soc. 23:1469-1475. https://doi.org/10.1590/S0103-50532012005000009

[41] Okada Y, Hihara T, Hirose M, Morita Z (2010) Substituent effects on the photofading of disperse azo dyes on poly (ethylene terephthalate) substrate. Color. Technol. 126:127-139. https://doi.org/10.1111/j.1478-4408.2010.00237.x 


\begin{tabular}{|c|c|c|c|c|c|c|c|c|}
\hline Functional & N1-C4 & C1-C2 & C2-C3 & C3-C4 & C4-C5 & $\mathrm{C} 5-\mathrm{C} 6$ & $\mathrm{C} 7-\mathrm{N} 2$ & N1-N2 \\
\hline \multicolumn{9}{|c|}{ Azobenzene } \\
\hline B3LYP & 1.41857 & 1.39555 & 1.39370 & 1.40115 & 1.40565 & 1.38951 & 1.41857 & 1.26056 \\
\hline CAM-B3LYP & 1.42042 & 1.38960 & 1.38894 & 1.39271 & 1.39728 & 1.38491 & 1.42042 & 1.24768 \\
\hline$\omega \mathrm{B} 97 \mathrm{XD}$ & 1.42240 & 1.39134 & 1.39070 & 1.39406 & 1.39883 & 1.38684 & 1.42240 & 1.24845 \\
\hline LC- $\omega \mathrm{PBE}$ & 1.42517 & 1.3801 & 1.38694 & 1.38846 & 1.39284 & 1.38322 & 1.42517 & 1.23960 \\
\hline Experimental [37] & 1.428 & 1.382 & 1.384 & 1.387 & 1.389 & 1.384 & 1.428 & 1.247 \\
\hline \multicolumn{9}{|c|}{ Disperse red 1} \\
\hline B3LYP & 1.39416 & 1.42078 & 1.38350 & 1.40472 & 1.41097 & 1.37881 & 1.41286 & 1.27051 \\
\hline CAM-B3LYP & 1.39957 & 1.41291 & 1.38044 & 1.39475 & 1.40131 & 1.37548 & 1.418431 & 1.25403 \\
\hline$\omega \mathrm{B} 97 \mathrm{XD}$ & 1.40155 & 1.41460 & 1.38211 & 1.39591 & 1.40246 & 1.37715 & 1.42071 & 1.2570 \\
\hline LC- $\omega \mathrm{PBE}$ & 1.40654 & 1.40912 & 1.37967 & 1.38982 & 1.39617 & 1.37465 & 1.42412 & 1.24435 \\
\hline \multicolumn{9}{|c|}{ Disperse red 73} \\
\hline B3LYP & 1.39141 & 1.41930 & 1.38270 & 1.40567 & 1.41178 & 1.37878 & 1.40504 & 1.27143 \\
\hline CAM-B3LYP & 1.39681 & 1.41139 & 1.37961 & 1.39572 & 1.40190 & 1.37564 & 1.41122 & 1.25479 \\
\hline$\omega \mathrm{B} 97 \mathrm{XD}$ & 1.398881 & 1.41288 & 1.38128 & 1.39681 & 1.40309 & 1.37732 & 1.41300 & 1.25527 \\
\hline LC- $\omega$ PBE & 1.40426 & 1.40747 & 1.37887 & 1.39067 & 1.39637 & 1.37502 & 1.41759 & 1.24476 \\
\hline Experimental [38] & 1.387 & 1.409 & 1.373 & 1.402 & 1.405 & 1.369 & 1.414 & 1.272 \\
\hline Functional & $\mathrm{N} 3-\mathrm{C} 1$ & $C 7-C 8$ & $\mathrm{Cl}_{-} \mathrm{CO}$ & $\mathrm{CO}-\mathrm{C} 10$ & C10_C11 & C11_C12 & $\mathrm{C} 12_{-} \mathrm{C} 7$ & C10-N4 \\
\hline \multicolumn{9}{|c|}{ Azobenzene } \\
\hline B3LYP & - & 1.40565 & 1.38951 & 1.40080 & 1.39555 & 1.39370 & 1.40115 & - \\
\hline CAM-B3LYP & - & 1.39728 & 1.38491 & 1.39454 & 1.38960 & 1.38894 & 1.39271 & - \\
\hline$\omega \mathrm{B} 97 \mathrm{XD}$ & - & 1.39883 & 1.38684 & 1.39598 & 1.39134 & 1.39070 & 1.39406 & - \\
\hline LC- $\omega$ PBE & - & 1.39284 & 1.38322 & 1.39156 & 1.38701 & 1.38694 & 1.38846 & - \\
\hline \multicolumn{9}{|c|}{ Disperse red 1} \\
\hline B3LYP & 1.37601 & 1.40919 & 1.38569 & 1.39866 & 1.39462 & 1.38883 & 1.40479 & 1.46574 \\
\hline CAM-B3LYP & 1.37250 & 1.39947 & 1.38204 & 1.39059 & 1.38668 & 1.38517 & 1.39511 & 1.46404 \\
\hline$\omega \mathrm{B} 97 \mathrm{XD}$ & 1.37210 & 1.40070 & 1.38394 & 1.39212 & 1.38843 & 1.38696 & 1.39631 & 1.46611 \\
\hline LC- $\omega$ PBE & 1.37047 & 1.39437 & 1.38079 & 1.38652 & 1.38277 & 1.38385 & 1.39044 & 1.46705 \\
\hline \multicolumn{9}{|c|}{ Disperse red 73} \\
\hline B3LYP & 1.46903 & 1.40685 & 1.38502 & 1.39868 & 1.38910 & 1.39930 & 1.41760 & 1.46903 \\
\hline CAM-B3LYP & 1.37657 & 1.39726 & 1.38148 & 1.39055 & 1.38214 & 1.39327 & 1.40567 & 1.46622 \\
\hline$\omega \mathrm{B} 97 \mathrm{XD}$ & 1.37624 & 1.39825 & 1.38338 & 1.39211 & 1.38414 & 1.39478 & 1.40632 & 1.46841 \\
\hline LC- $\omega$ PBE & 1.37490 & 1.39214 & 1.38033 & 1.38639 & 1.37899 & 1.39032 & 1.39926 & 1.46855 \\
\hline Experimental [38] & 1.357 & 1.391 & 1.379 & 1.386 & 1.375 & 1.400 & 1.402 & 1.472 \\
\hline
\end{tabular}




\begin{tabular}{|c|c|c|c|c|c|}
\hline \multicolumn{7}{|c|}{ Table 1(b): Optimized bond angles of molecules using 6-31G(d,p) basis set } \\
\hline Functional & C3-C4-N1 & C5-C4-N1 & C8-C7-N2 & C12-C7-N2 & C4-N1-N2-C7 \\
\hline B3LYP & 115.334 & 124.776 & 124.776 & 115.334 & 180.00 \\
\hline CAM-B3LYP & 115.4410 & 124.412 & 124.412 & 115.441 & 180.000 \\
\hline$\omega$ B97XD & 115.344 & 124.443 & 124.443 & 115.344 & 180.000 \\
\hline LC- $\omega$ PBE & 115.530 & 124.128 & 124.128 & 115.530 & 180.000 \\
\hline Experimental [37] & 120.7 & 123.7 & & \\
\hline \multicolumn{7}{|c|}{ Disperse Red 1 } \\
\hline B3LYP & 116.394 & 125.529 & 124.904 & 115.496 & -179.953 \\
\hline CAM-B3LYP & 116.458 & 125.127 & 124.440 & 115.501 & -179.942 \\
\hline$\omega$ B97XD & 116.340 & 125.156 & 124.523 & 115.379 & -179.957 \\
\hline LC- $\omega$ PBE & 116.537 & 124.903 & 124.145 & 115.523 & -179.953 \\
\hline \multicolumn{7}{|c|}{ Disperse Red 73 } \\
\hline B3LYP & 116.405 & 125.210 & 125.147 & 115.684 & 179.935 \\
\hline CAM-B3LYP & 116.474 & 124.797 & 124.738 & 115.691 & 179.967 \\
\hline$\omega$ B97XD & 116.372 & 124.782 & 124.905 & 115.503 & -180.00 \\
\hline LC- $\omega$ PBE & 116.537 & 124.572 & 124.486 & 115.722 & 179.959 \\
\hline Experimental [38] & 115.8 & 126.2 & 124.8 & 116.5 & - \\
\hline
\end{tabular}

\begin{tabular}{|c|c|c|c|c|c|}
\hline \multicolumn{5}{|c|}{ Table 1(c): Absolute average error for C-C, C-N, N-N and C-C-N bond measurements } \\
\hline Molecule & Bond Length/Angle & B3LYP & CAM-B3LYP & $\omega$ B97XD & LC- $\omega$ PBE \\
\hline Azobenzene & 5 C-C & 0.0119 & 0.0055 & 0.0072 & 0.0011 \\
& $2 \mathrm{C}-\mathrm{N}$ & 0.0094 & 0.0076 & 0.0056 & 0.0028 \\
& $1 \mathrm{~N}-\mathrm{N}$ & 0.014 & 0.001 & 0.002 & 0.007 \\
& $2 \mathrm{C}-\mathrm{C}-\mathrm{N}$ & 2.145 & 2.2735 & 2.3065 & 2.3571 \\
\hline Disperse Red 73 & $11 \mathrm{C}-\mathrm{C}$ & 0.0094 & 0.0021 & 0.036 & 0.0014 \\
& $4 \mathrm{C}-\mathrm{N}$ & 0.0308 & 0.0048 & 0.0086 & 0.0090 \\
& $1 \mathrm{~N}-\mathrm{N}$ & 0.002 & 0.015 & 0.015 & 0.025 \\
& $4 \mathrm{C}-\mathrm{C}-\mathrm{N}$ & 0.214 & 0.400 & 0.435 & 0.496 \\
\hline
\end{tabular}

\begin{tabular}{|c|c|c|c|c|c|c|c|c|}
\hline & & $\begin{array}{c}\text { Table } 2 \\
0\rangle \rightarrow|2\rangle\end{array}$ & $\begin{array}{l}\text { Excit } \\
\text { ransiti }\end{array}$ & $\begin{array}{l}\text { on eners } \\
\text { of targe }\end{array}$ & $\begin{array}{l}\text { and Tr } \\
\text { molecul }\end{array}$ & $\begin{array}{l}\text { sition di } \\
\text { using } 6 \text { - }\end{array}$ & $\begin{array}{l}\text { le moments of } \\
G(d, p) \text { basis set }\end{array}$ & \\
\hline Functional & $\lambda(\exp )$ & $\lambda$ & $\mathrm{E}$ & $\mu_{x}$ & $\mu_{y}$ & $\mu_{z}$ & Dipole Strength & Oscillator Strength (f) \\
\hline & $(\mathrm{nm})$ & $(\mathrm{nm})$ & $\mathrm{eV}$ & & & & & \\
\hline & & & & Azob & zzene $\mathrm{mc}$ & cule & & \\
\hline B3LYP & 325 [39] & 328.93 & 3.7693 & 0.1223 & -2.8896 & 0 & 8.3645 & .7724 \\
\hline CAM-B3LYP & 325 [39] & 303.77 & 4.0815 & -0.0761 & 2.8521 & 0 & 8.1402 & 0.8140 \\
\hline LC- $\omega \mathrm{PBE}$ & 325 [39] & 383.66 & 4.3709 & -0.0557 & 2.7614 & 0 & 7.6283 & 0.8169 \\
\hline$\omega \mathrm{B} 97 \mathrm{XD}$ & 325 [39] & 302.79 & 4.097 & -0.0757 & 2.8505 & 0 & 8.1312 & 0.8157 \\
\hline & & & & Dispers & Red 1 1 & lecule & & \\
\hline B3LYP & $406[40]$ & 471.04 & 2.6321 & -3.7996 & 0.0741 & -0.0828 & 14.449 & 0.9317 \\
\hline CAM-B3LYP & $406[40]$ & 395.90 & 3.1317 & -4.0868 & 0.0527 & -0.0964 & 16.7137 & 1.2823 \\
\hline LC- $\omega \mathrm{PBE}$ & $406[40]$ & 361.33 & 3.4313 & -3.9788 & 0.0328 & -0.0992 & 15.8418 & 1.3318 \\
\hline$\omega \mathrm{B} 97 \mathrm{XD}$ & $406[40]$ & 387.91 & 3.1962 & -4.0940 & 0.0504 & -0.0979 & 16.7726 & 1.3134 \\
\hline & & & & Dispers & Red 73 & plecule & & \\
\hline B3LYP & $521[41]$ & 470.81 & 2.6334 & -0.0096 & 0.0038 & -0.0262 & 14.7062 & 0.9488 \\
\hline CAM-B3LYP & $521[41]$ & 399.65 & 3.1023 & -4.0365 & -0.3462 & 0.0173 & 16.4138 & 1.2475 \\
\hline LC- $\omega$ PBE & 521 [41] & 365.96 & 3.3879 & -3.9220 & -0.3600 & 0.0132 & 15.5120 & 1.2875 \\
\hline$\omega \mathrm{B} 97 \mathrm{XD}$ & 521 [41] & 391.83 & 3.1642 & -4.0368 & -0.3534 & 0.0160 & 16.4209 & 1.2730 \\
\hline
\end{tabular}




\begin{tabular}{|c|c|c|c|c|c|c|}
\hline \multicolumn{1}{|c|}{ Table 3 : Static molecular polarizability of target molecules using 6-31G(d,p) basis set } \\
\hline & Azobenzene molecule & \multicolumn{2}{c|}{ Disperse Red1 } & \multicolumn{2}{|c|}{ Disperse Red 73 } \\
\hline Functional & $\alpha$ & $\Delta \alpha$ & $\alpha$ & $\Delta \alpha$ & $\alpha$ & $\Delta \alpha$ \\
\hline & Bohr $^{3}$ & Bohr $^{3}$ & Bohr $^{3}$ & Bohr $^{3}$ & Bohr $^{3}$ & Bohr $^{3}$ \\
\hline B3LYP & 73.885 & 162.240 & 158.158 & 423.514 & 158.649 & 413.363 \\
\hline CAM-B3LYP & 68.269 & 144.113 & 135.644 & 346.781 & 139.720 & 341.363 \\
\hline LC- $\omega$ PBE & 63.424 & 129.558 & 120.100 & 297.924 & 125.212 & 294.637 \\
\hline$\omega$ B97XD & 68.326 & 143.616 & 133.039 & 338.396 & 137.193 & 332.577 \\
\hline
\end{tabular}




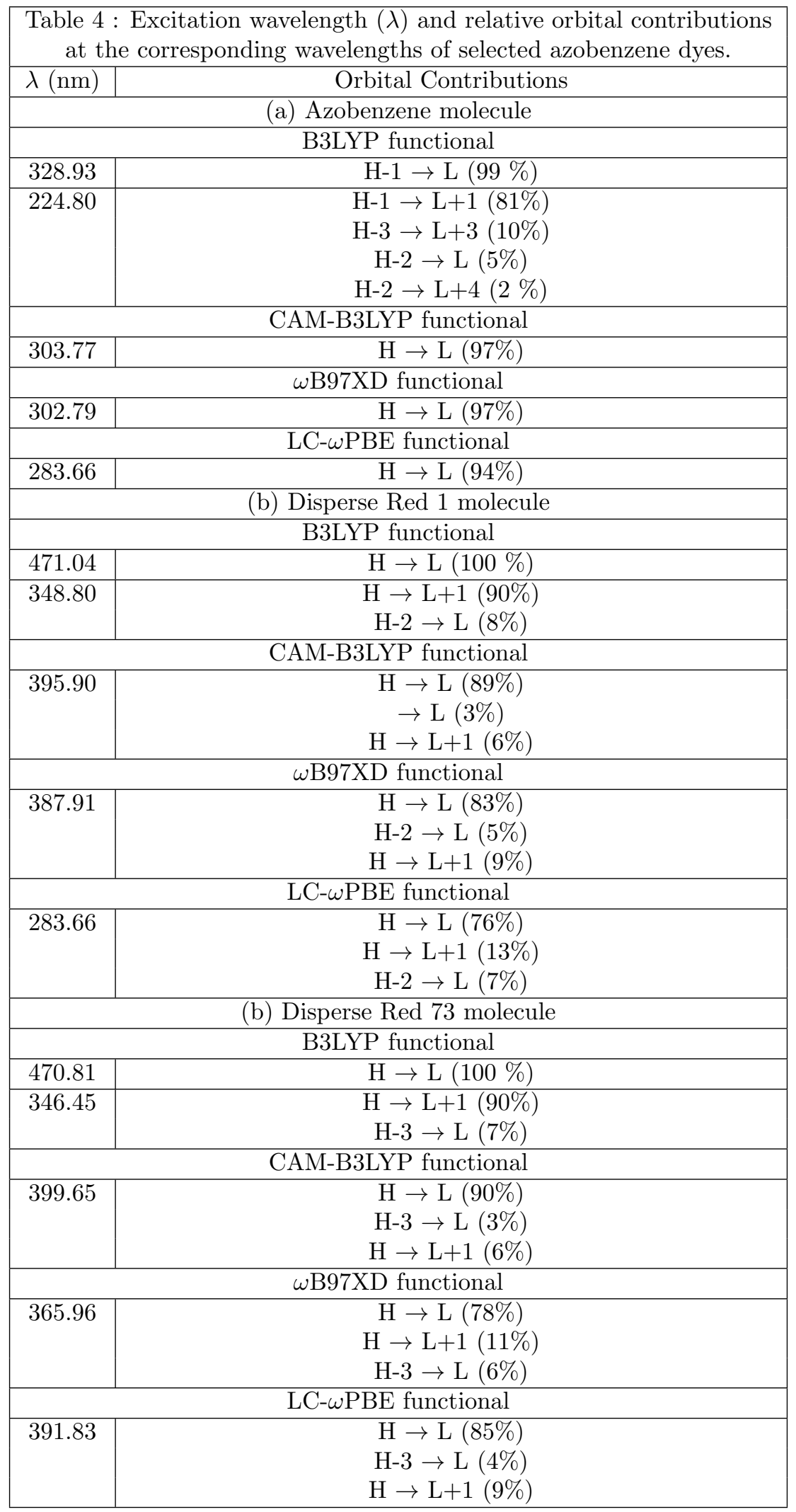

where $\mathrm{H}=\mathrm{HOMO}$ i.e Highest Occupied Molecular Orbital and $\mathrm{L}=\mathrm{LUMO}$ i.e. Lowest Unoccupied Molecular Orbital 


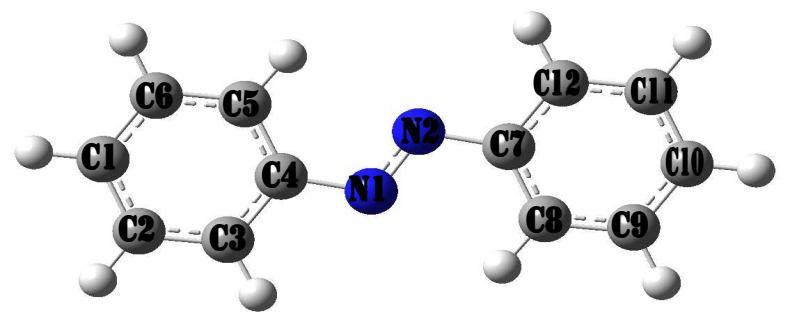

(a) Azobenzene

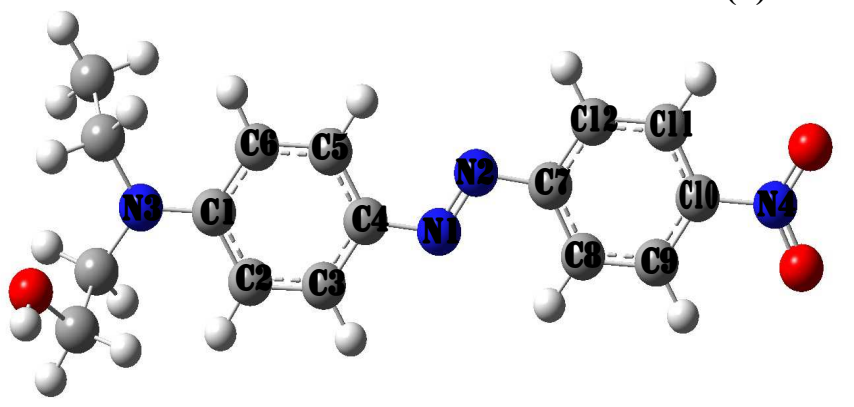

(b) Disperse Red 1

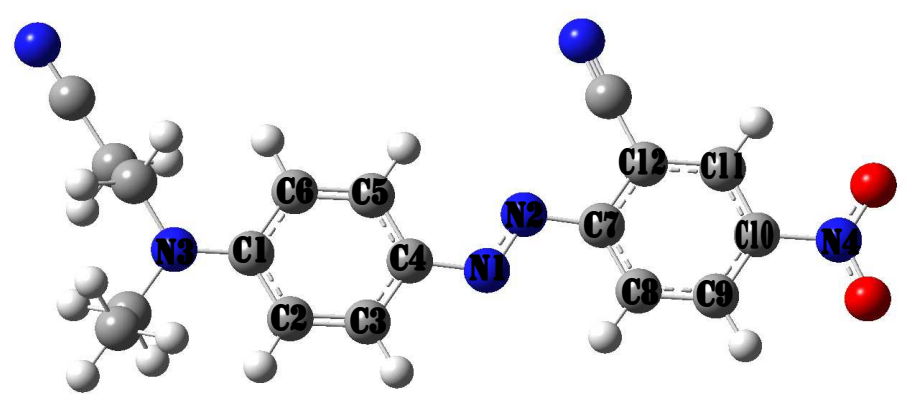

(c) Disperse Red 73

Figure 1: Molecular structure of (a) Azobenzene (b) Disperse Red 1 (c) Disperse Red 73 molecules
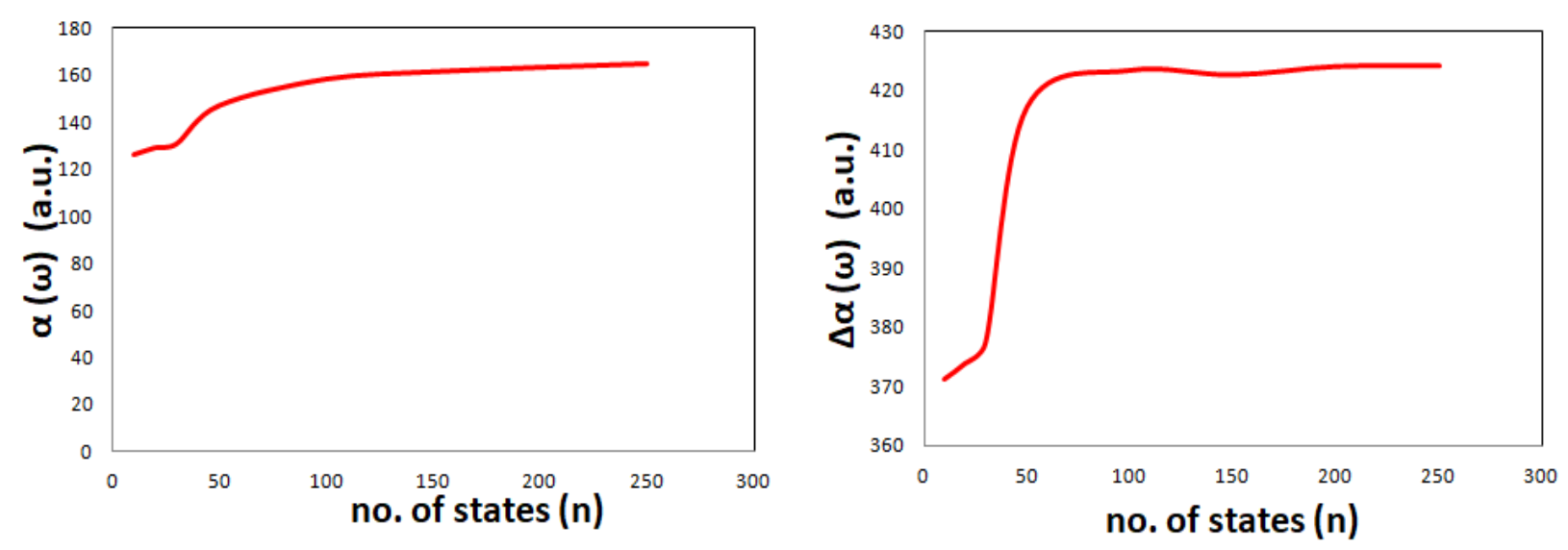

Figure 2: Plot of isotropic $(\alpha(\omega))$ and anisotropic $(\Delta \alpha(\omega))$ polarizability of Azobenzene molecule as a function of number of states (n) calculated using B3LYP functional and 6-31G(d,p) basis set. 


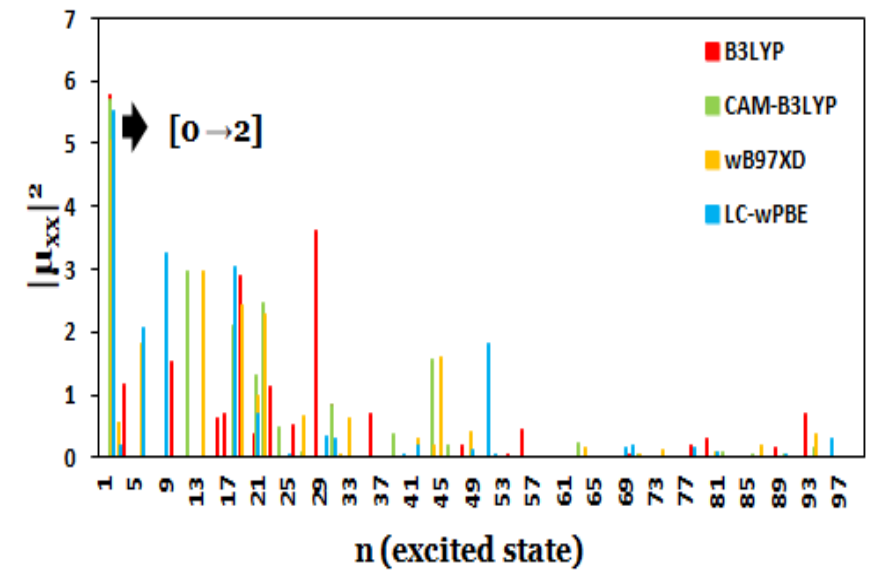

(a) Azobenzene

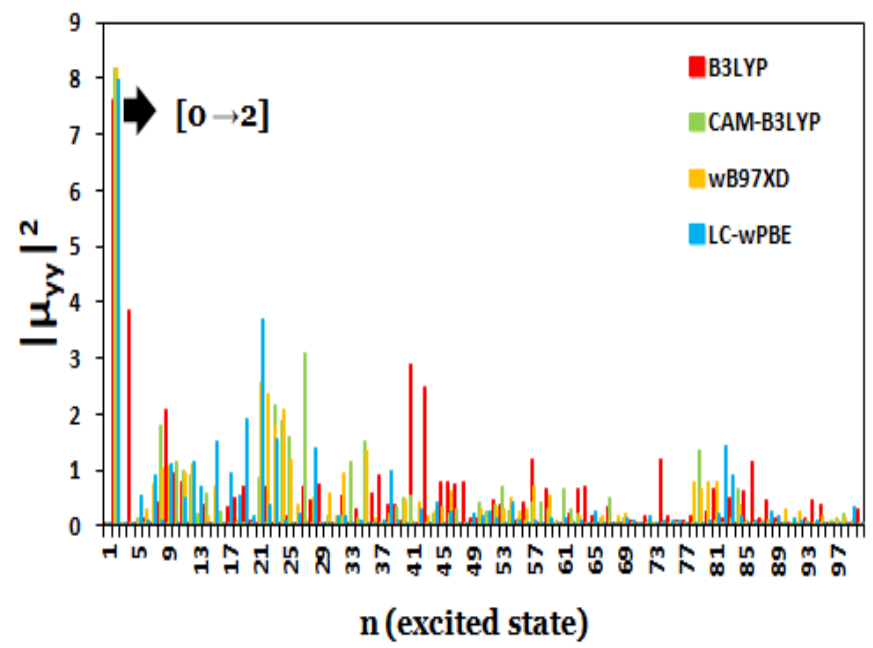

(b) Disperse Red 1

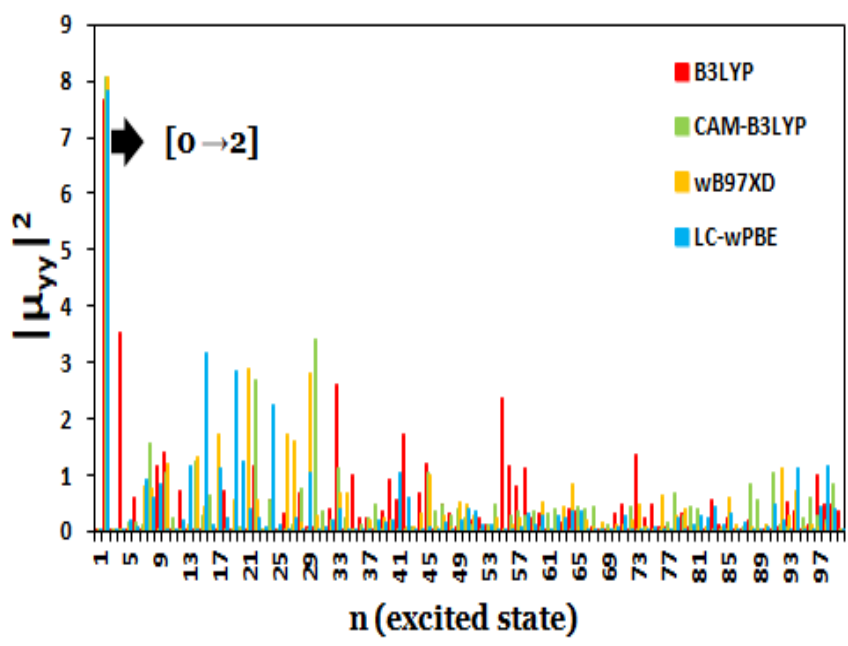

(c) Disperse Red 73

Figure 3: Transition dipole moments of molecules (a) Azobenzene $\left(\left|\mu_{x x}^{2}\right|\right)$ (b) Disperse Red $1\left(\left|\mu_{y y}^{2}\right|\right)$

(c) Disperse Red $73\left(\left|\mu_{y y}^{2}\right|\right)$ for all 100 excited states contributing to maximum polarizability components. 


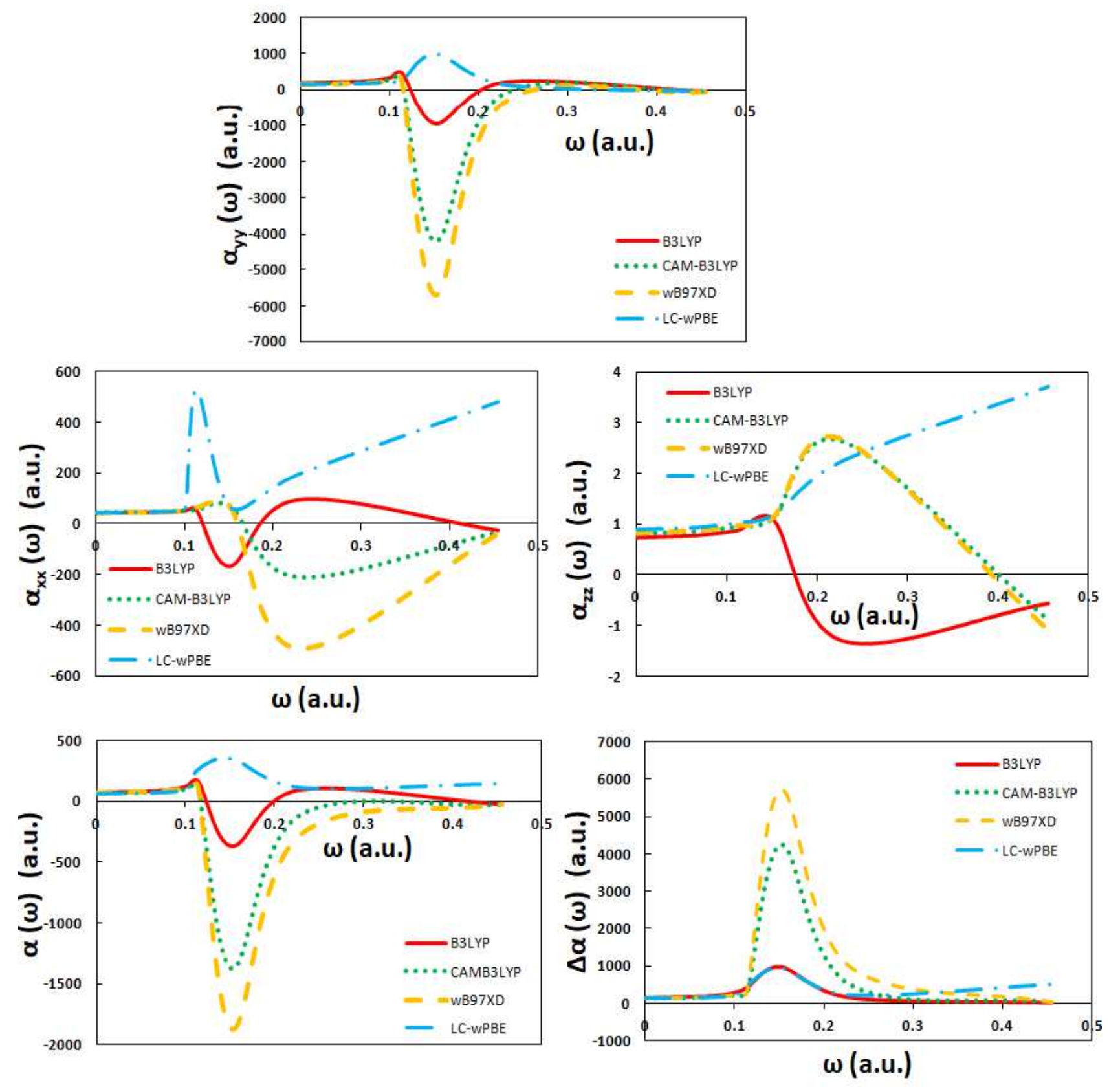

Figure 4: Plot of polarizability tensor components $\left(\alpha_{x x}(\omega), \alpha_{y y}(\omega)\right.$ and $\left.\alpha_{z z}(\omega)\right)$ and isotropic $(\alpha(\omega))$ and anisotropic $(\Delta \alpha(\omega))$ polarizability of Azobenzene molecule as a function of frequency $(\omega)$ of external electric field 

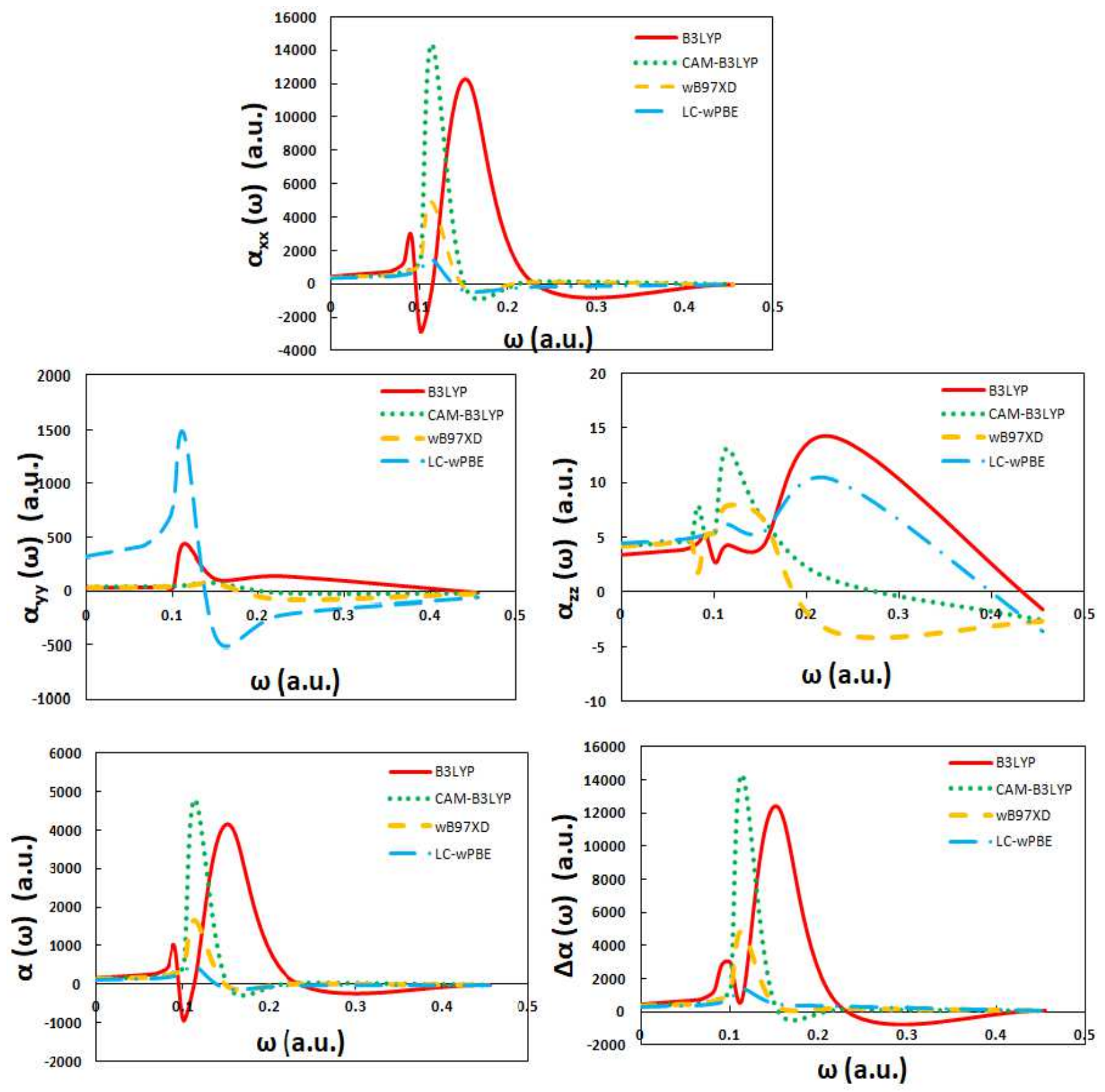

Figure 5: Plot of polarizability tensor components $\left(\alpha_{x x}(\omega), \alpha_{y y}(\omega)\right.$ and $\left.\alpha_{z z}(\omega)\right)$ and isotropic $(\alpha(\omega))$ and anisotropic $(\Delta \alpha(\omega))$ polarizability of Disperse Red 1 molecule as a function of frequency ( $\omega)$ of external electric field 

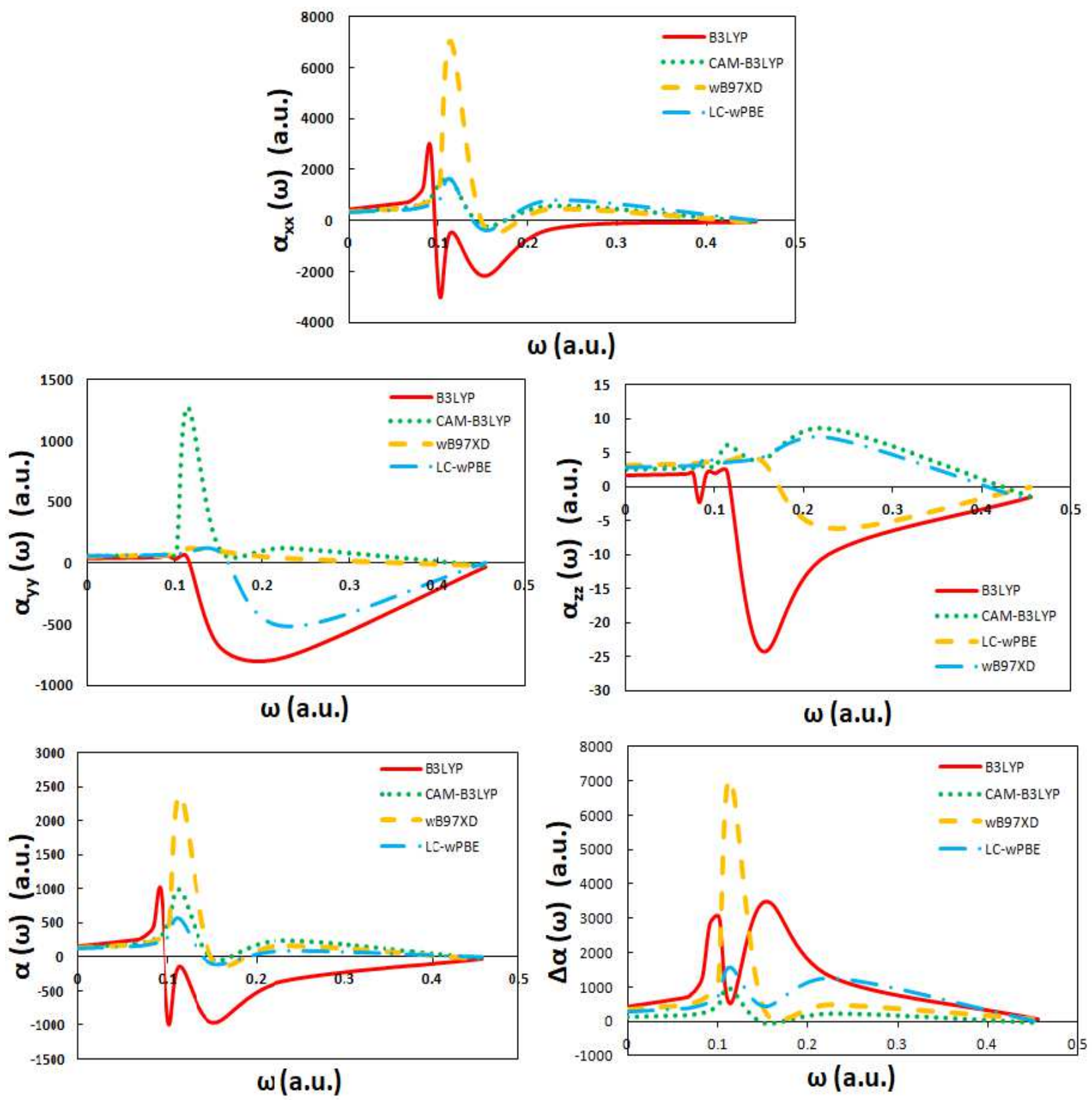

Figure 6: Plot of polarizability tensor components $\left(\alpha_{x x}(\omega), \alpha_{y y}(\omega)\right.$ and $\left.\alpha_{z z}(\omega)\right)$ and isotropic $(\alpha(\omega))$ and anisotropic $(\Delta \alpha(\omega))$ polarizability of Disperse Red 73 molecule as a function of frequency $(\omega)$ of external electric field 

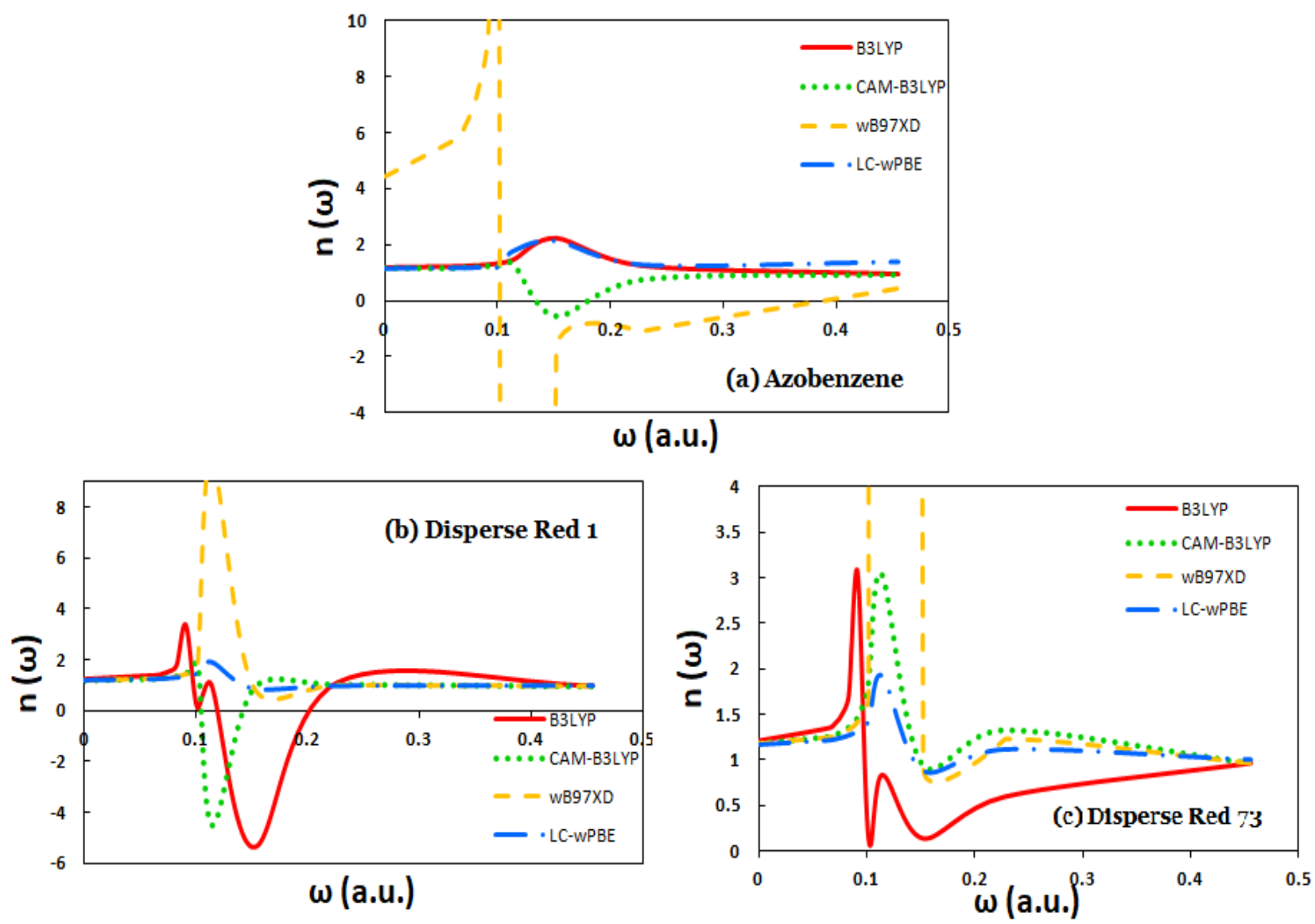

Figure 7: Refractive index $(n(\omega))$ of molecules (a) Azobenzene (b) Disperse Red 1 (c) Disperse Red 73 as a function of frequency $(\omega)$ of external electric field 


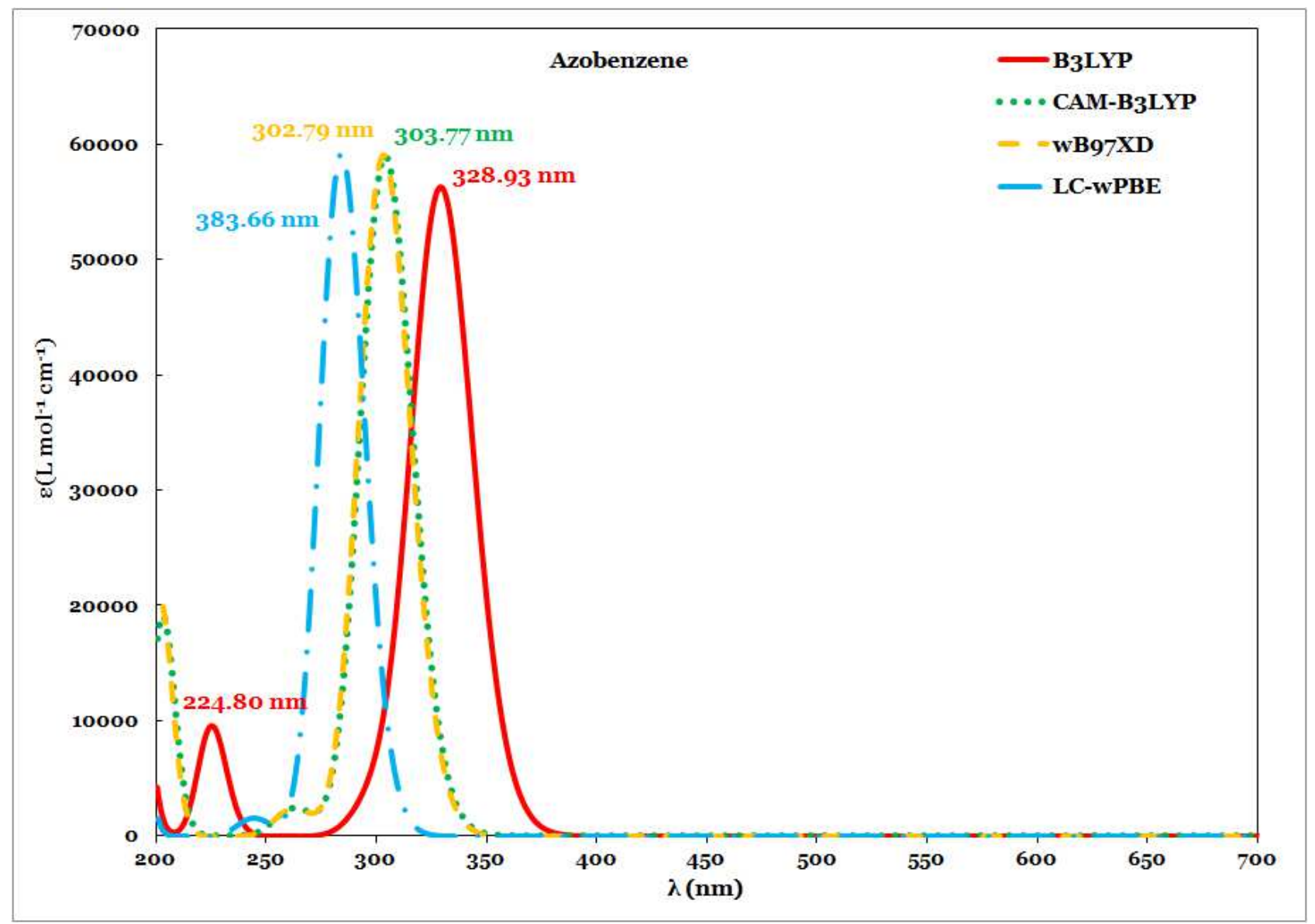

Figure 8: UV-Visible Spectra of Azobenzene molecule 


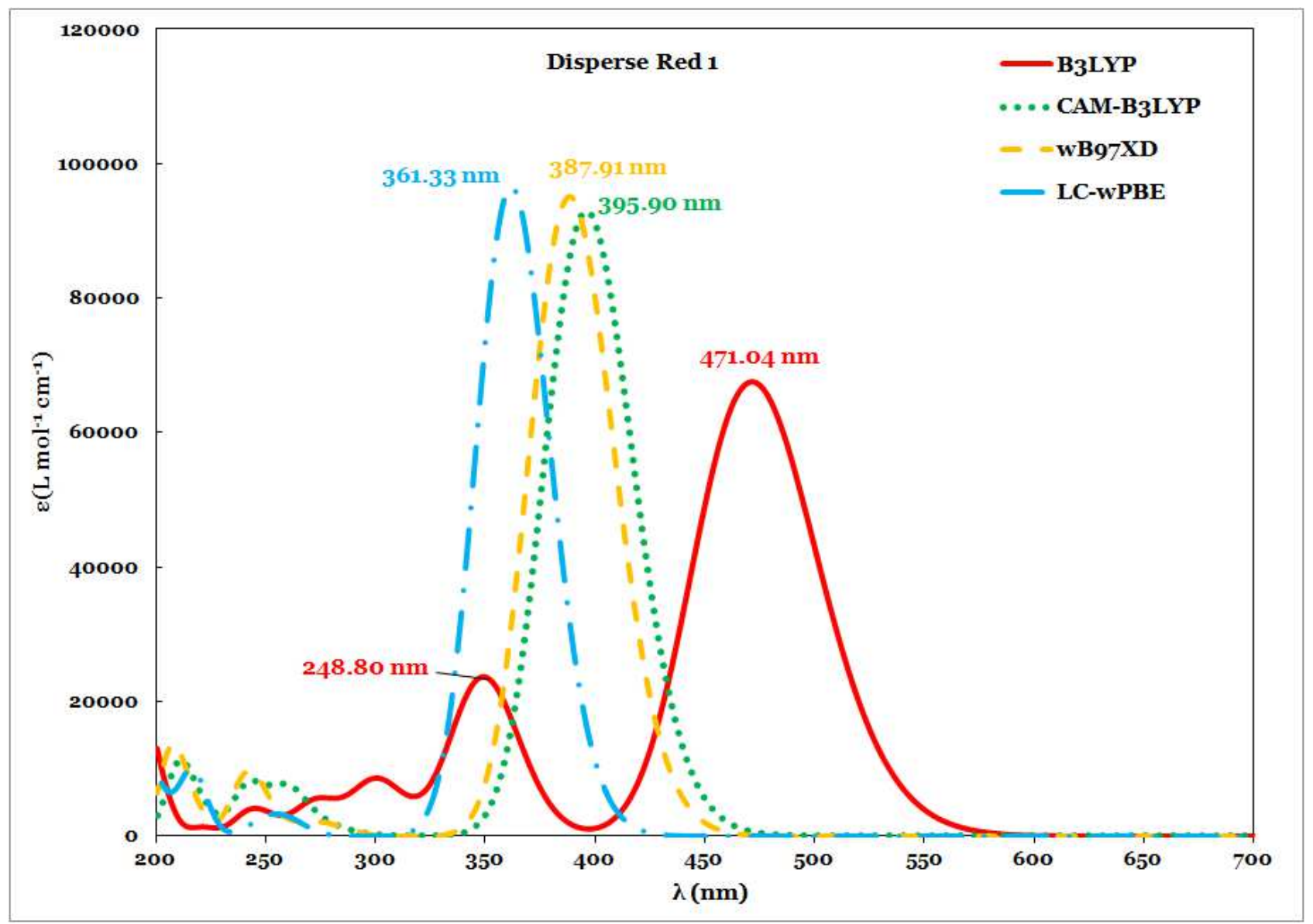

Figure 9: UV-Visible Spectra of Disperse Red 1 molecule 


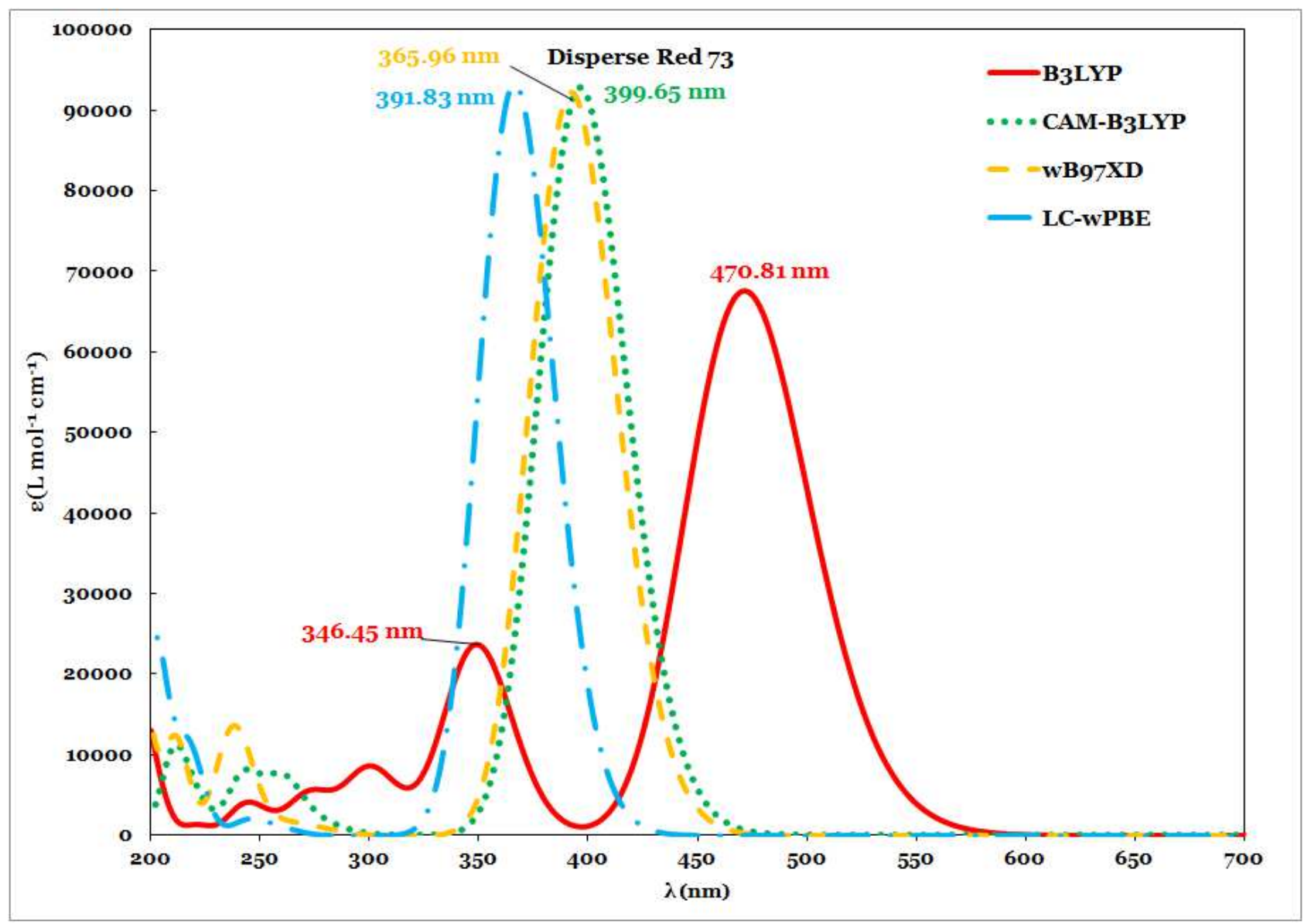

Figure 10: UV-Visible Spectra of Disperse Red 73 molecule 


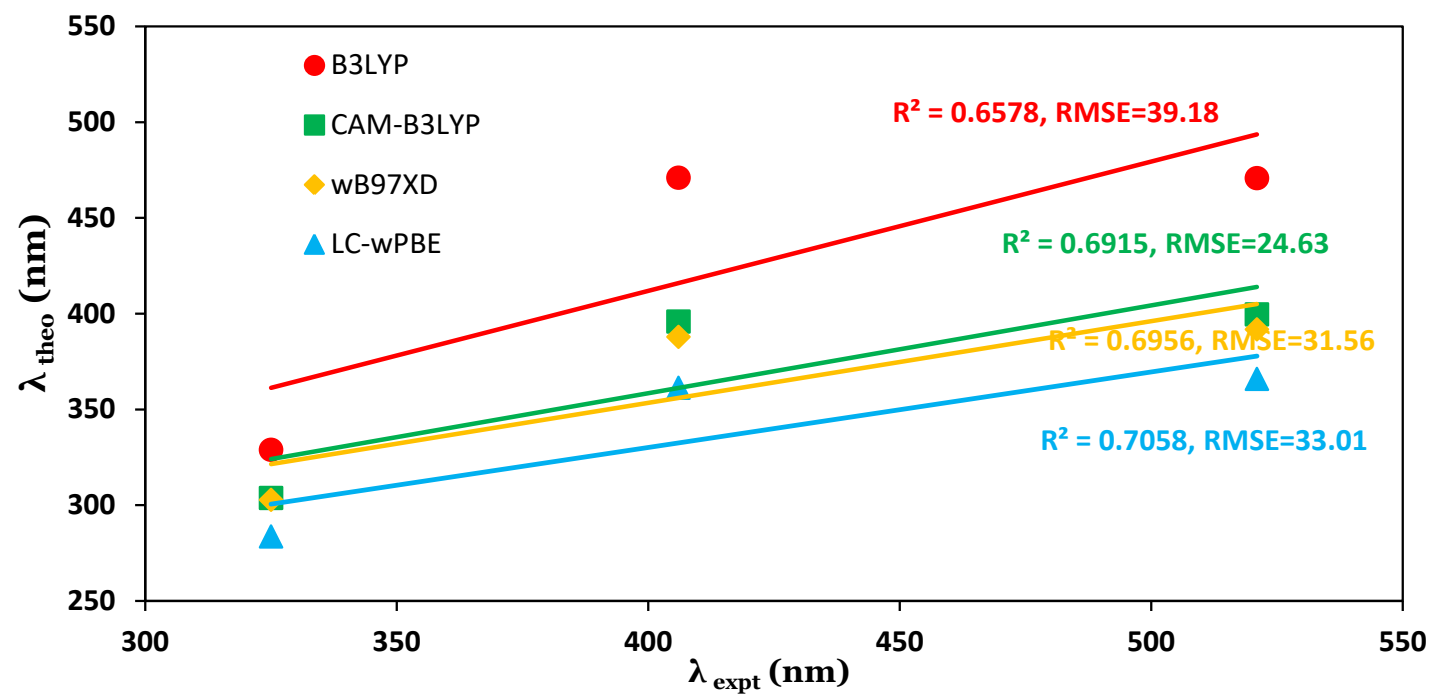

Figure 11: Comparison between theoretical $\left(\lambda_{\text {theo }}\right)$ and experimental wavelengths $\left(\lambda_{\text {expt }}\right)$ of $U V$-Visible spectra for all dye molecules (where $R^{2}$ is the correlation coefficient and RMSE is the Root Mean Square Error) 
Figures

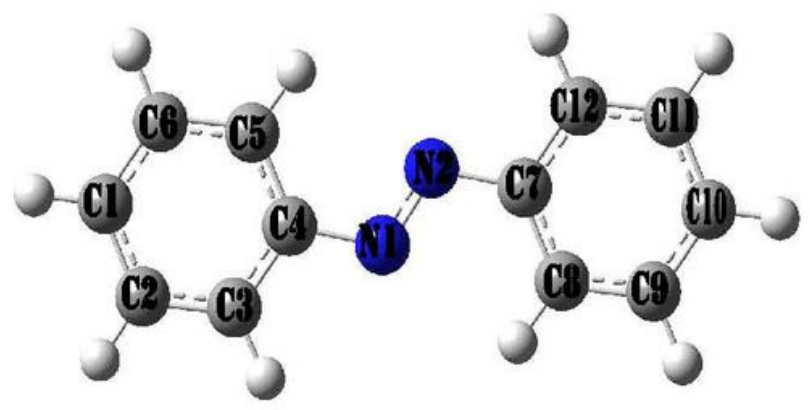

(a) Azobenzene

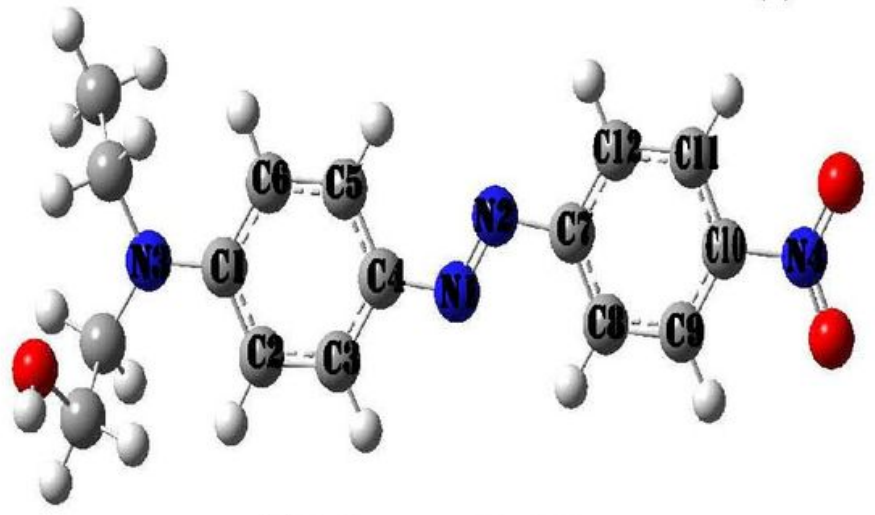

(b) Disperse Red 1

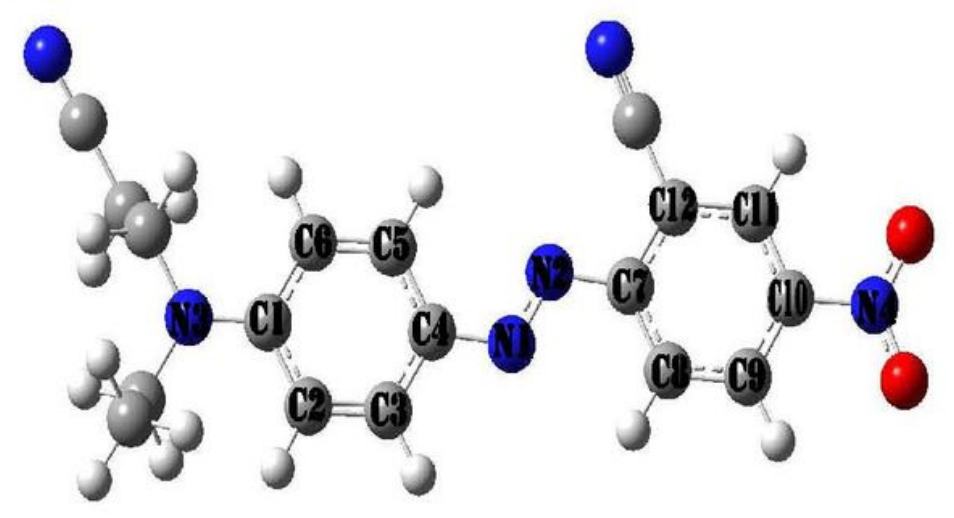

(c) Disperse Red 73

Figure 1

Molecular structure of (a) Azobenzene (b) Disperse Red 1 (c) Disperse Red 73 molecules
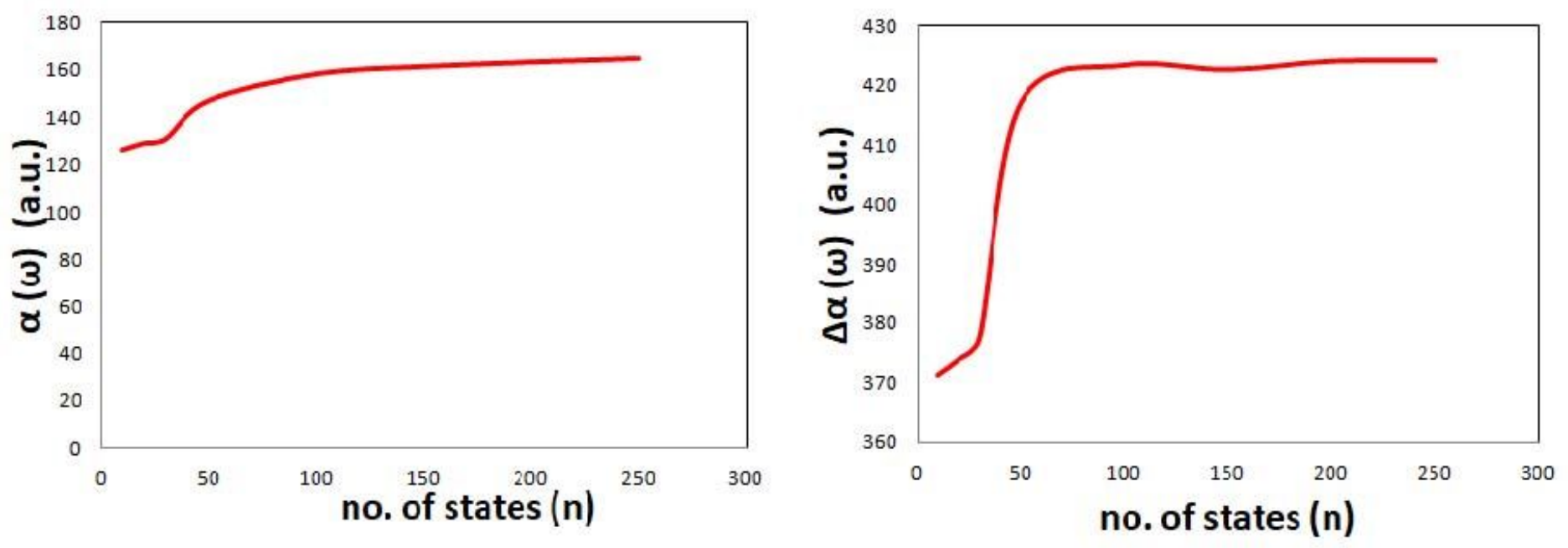

Figure 2

Plot of isotropic $(a(\omega))$ and anisotropic $(\Delta a(\omega))$ polarizability of Azobenzene molecule as a function of number of states $(n)$ calculated using B3LYP functional and 6-31G(d,p) basis set. 


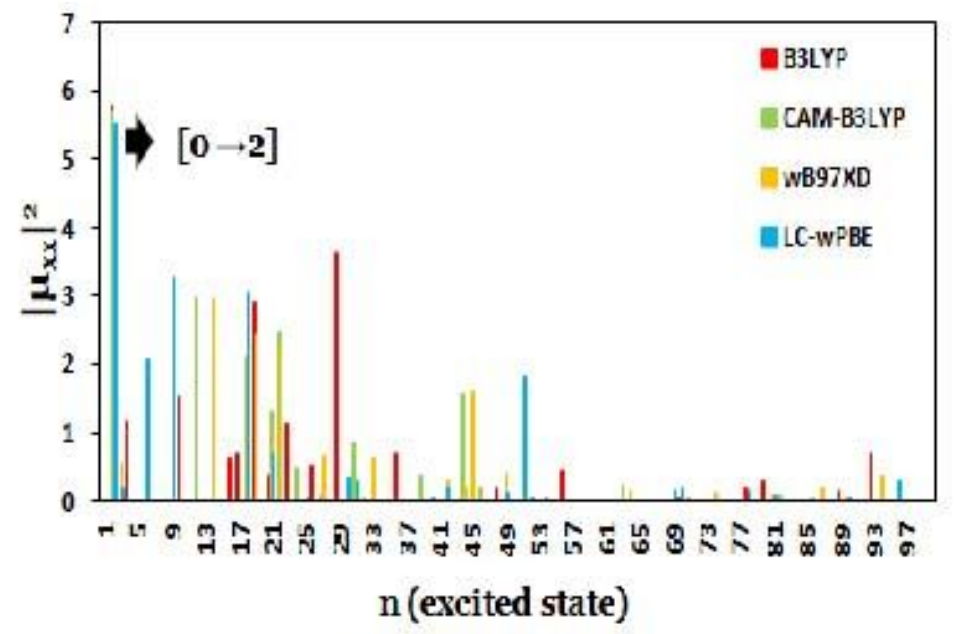

(a) Azobenzene

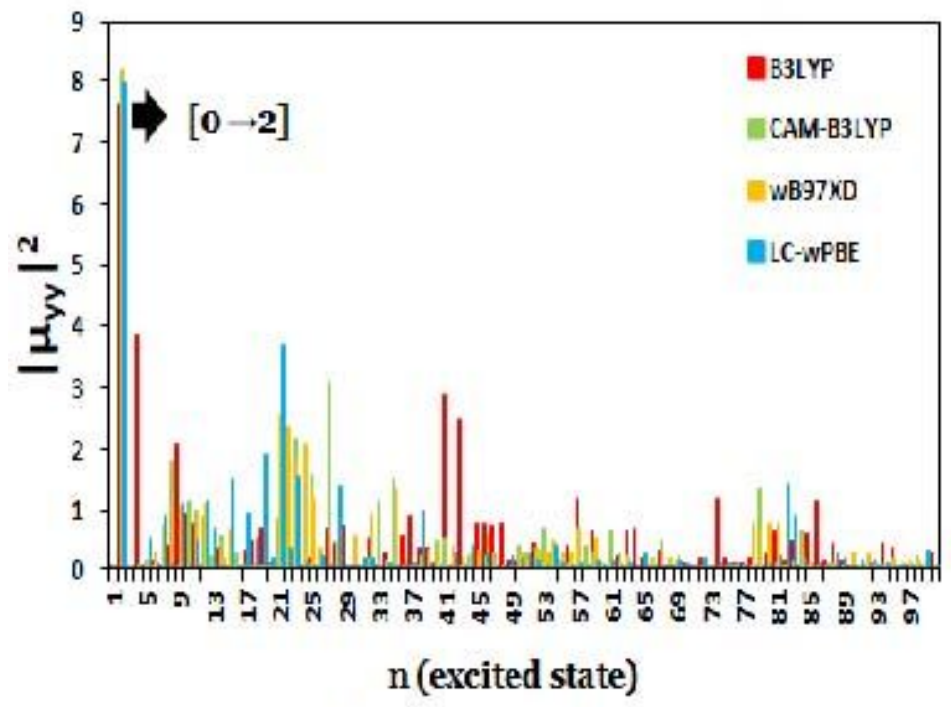

(b) Disperse Red 1

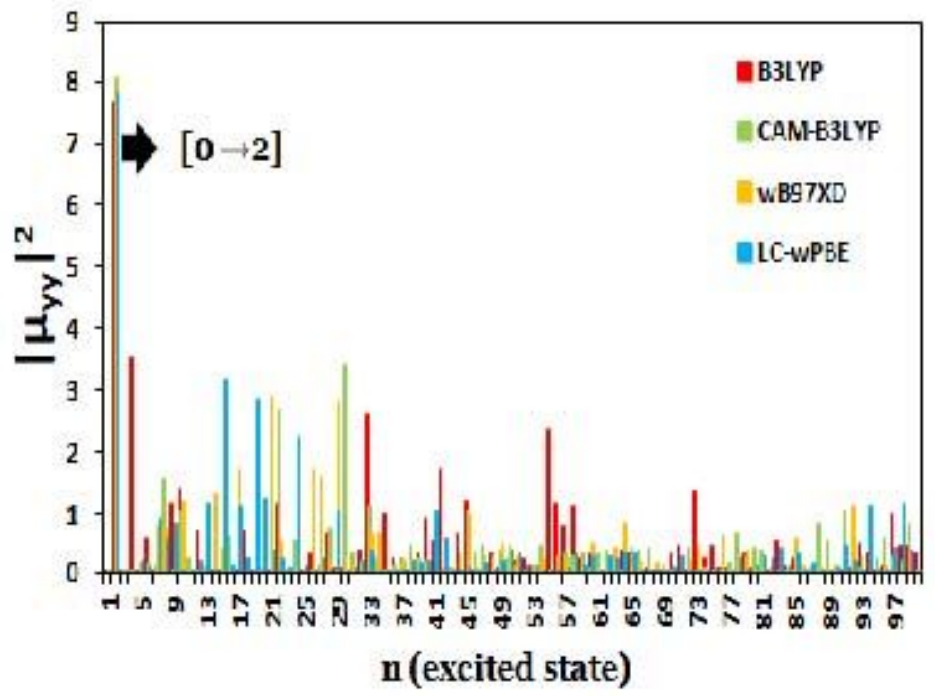

(c) Disperse Red 73

Figure 3

"Please see the Manuscript PDF file for the complete figure caption". 


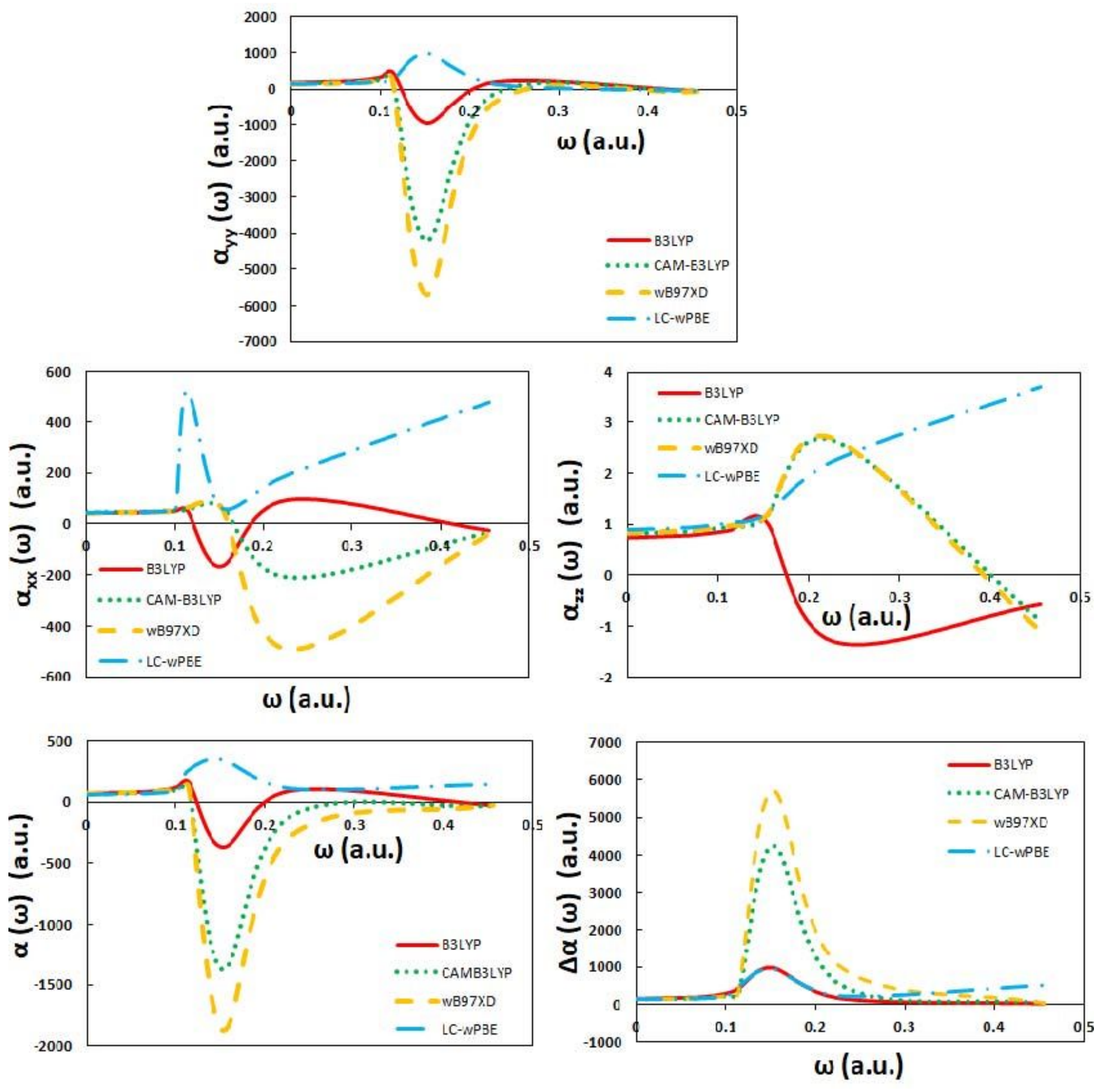

Figure 4

Plot of polarizability tensor components $(\operatorname{axx}(\omega)$, ayy $(\omega)$ and $\operatorname{azz}(\omega))$ and isotropic $(a(\omega))$ and anisotropic $(\Delta \mathrm{a}(\omega))$ polarizability of Azobenzene molecule as a function of frequency $(\omega)$ of external electric field 


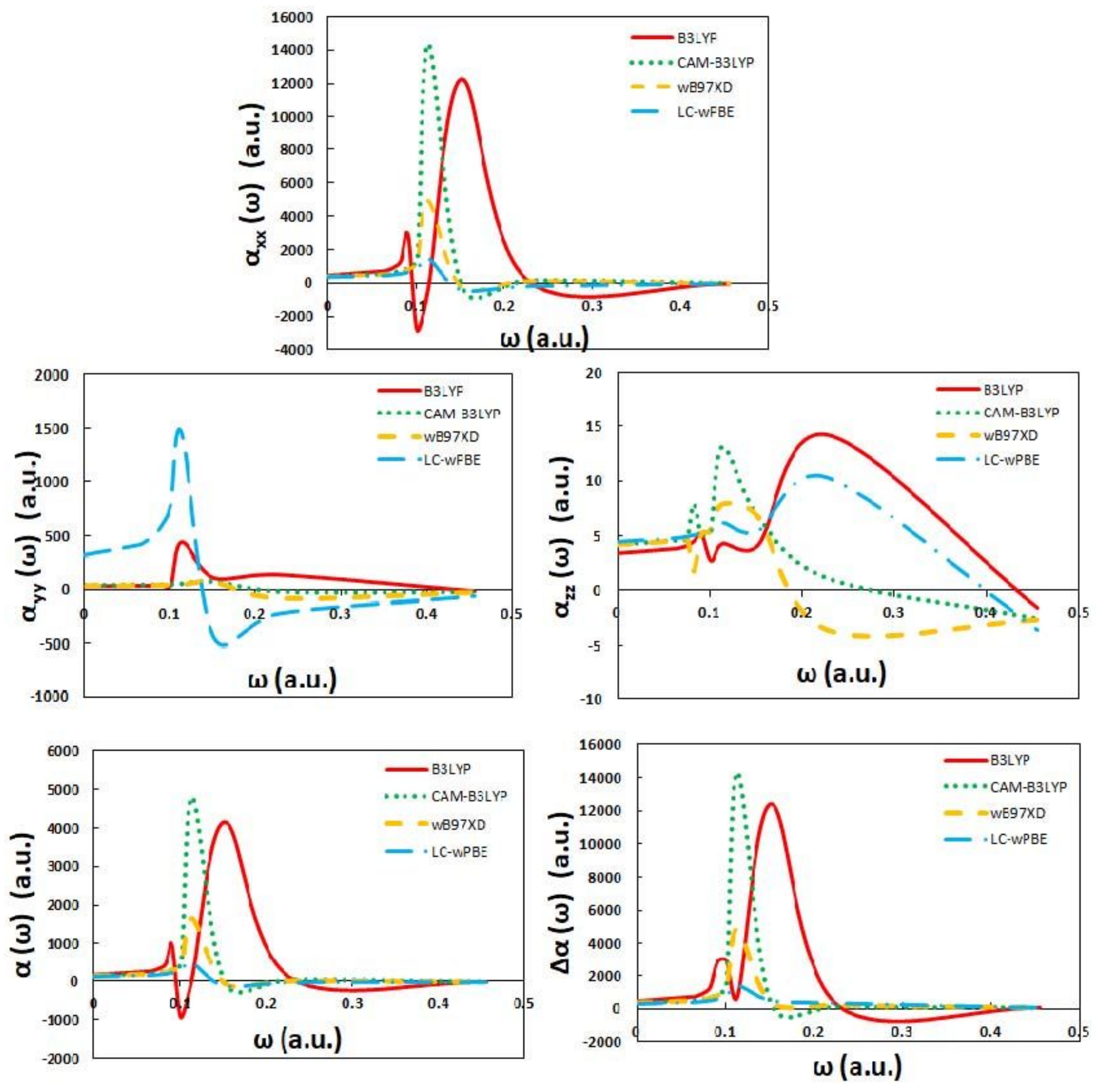

Figure 5

Plot of polarizability tensor components $(\operatorname{axx}(\omega)$, ayy $(\omega)$ and $\operatorname{azz}(\omega))$ and isotropic $(a(\omega))$ and anisotropic $(\Delta a(\omega))$ polarizability of Disperse Red 1 molecule as a function of frequency $(\omega)$ of external electric field 

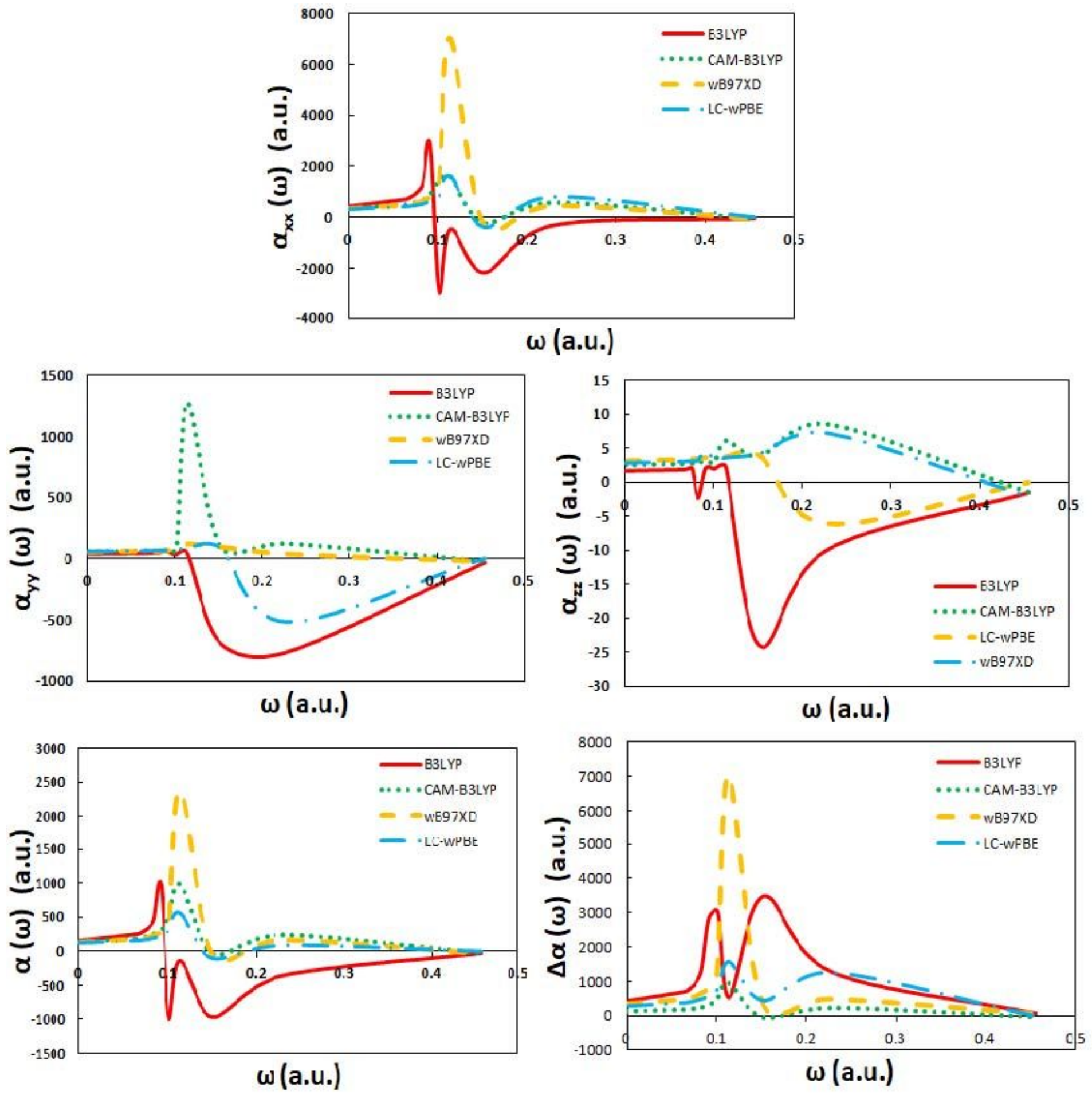

Figure 6

Plot of polarizability tensor components $(\operatorname{axx}(\omega)$, ayy $(\omega)$ and $\operatorname{azz}(\omega))$ and isotropic $(a(\omega))$ and anisotropic $(\Delta a(\omega))$ polarizability of Disperse Red 73 molecule as a function of frequency $(\omega)$ of external electric field 

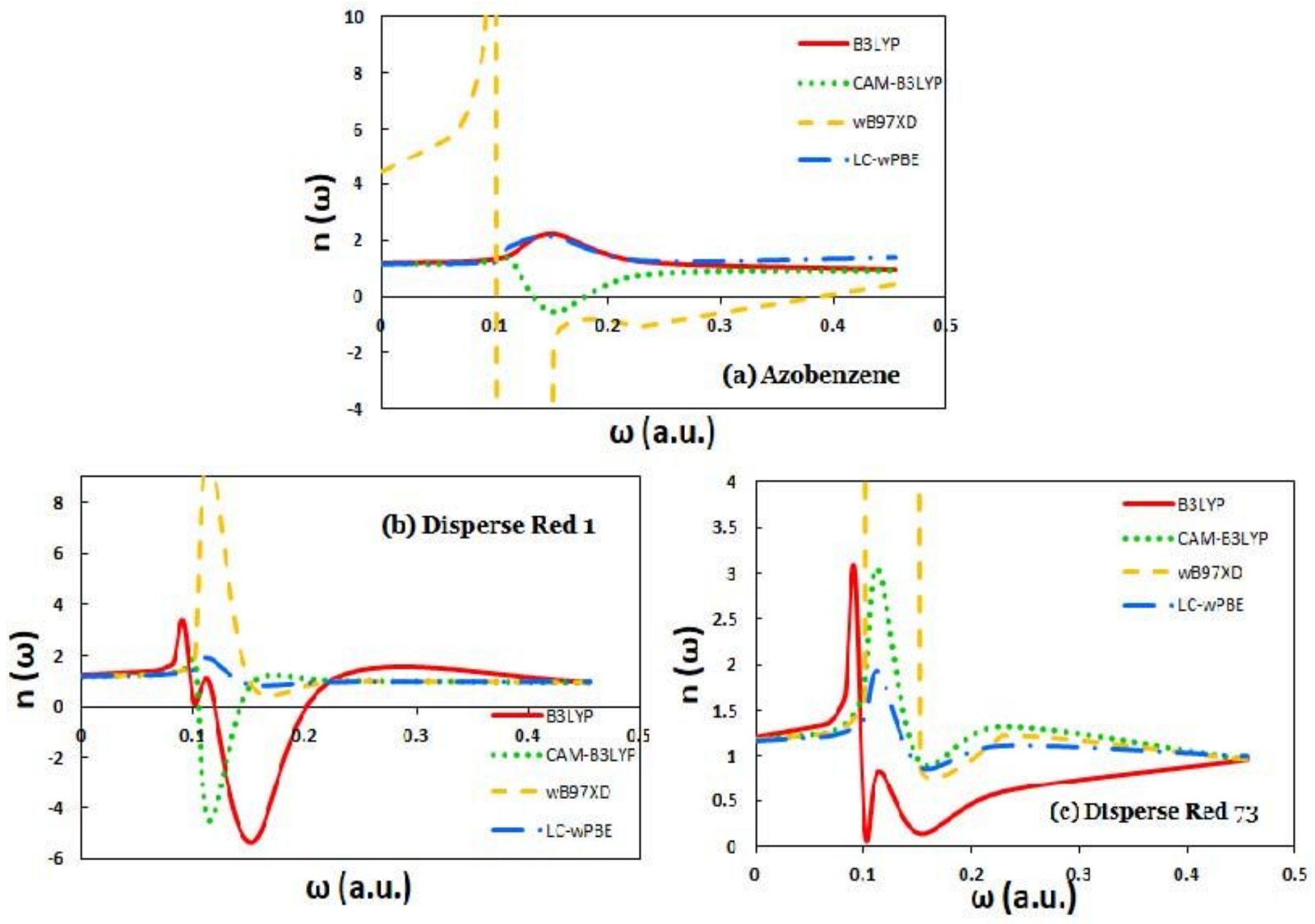

Figure 7

Refractive index ( $n(\omega))$ of molecules (a) Azobenzene (b) Disperse Red 1 (c) Disperse Red 73 as a function of frequency $(\omega)$ of external electric field 


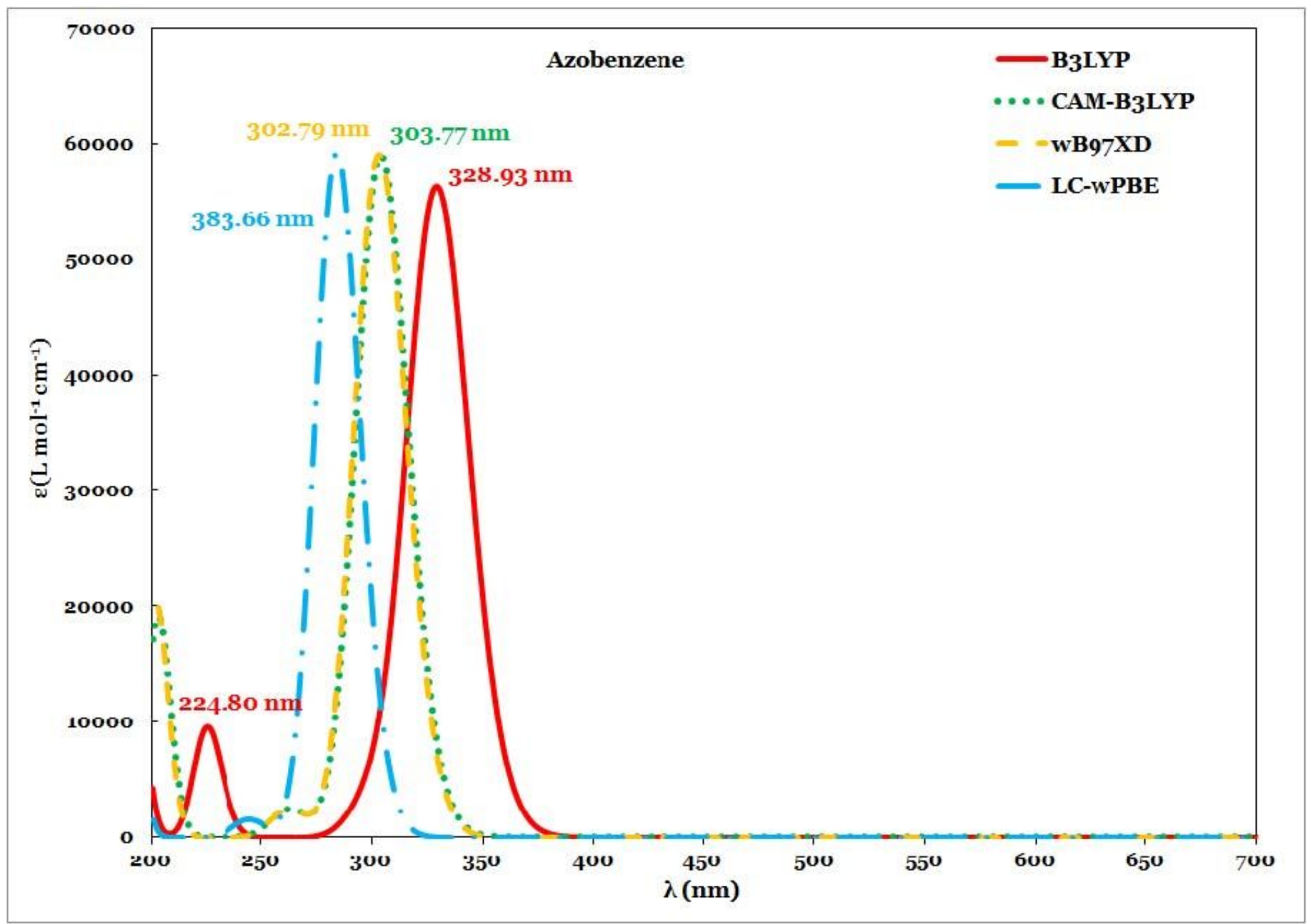

Figure 8

UV-Visible Spectra of Azobenzene molecule 


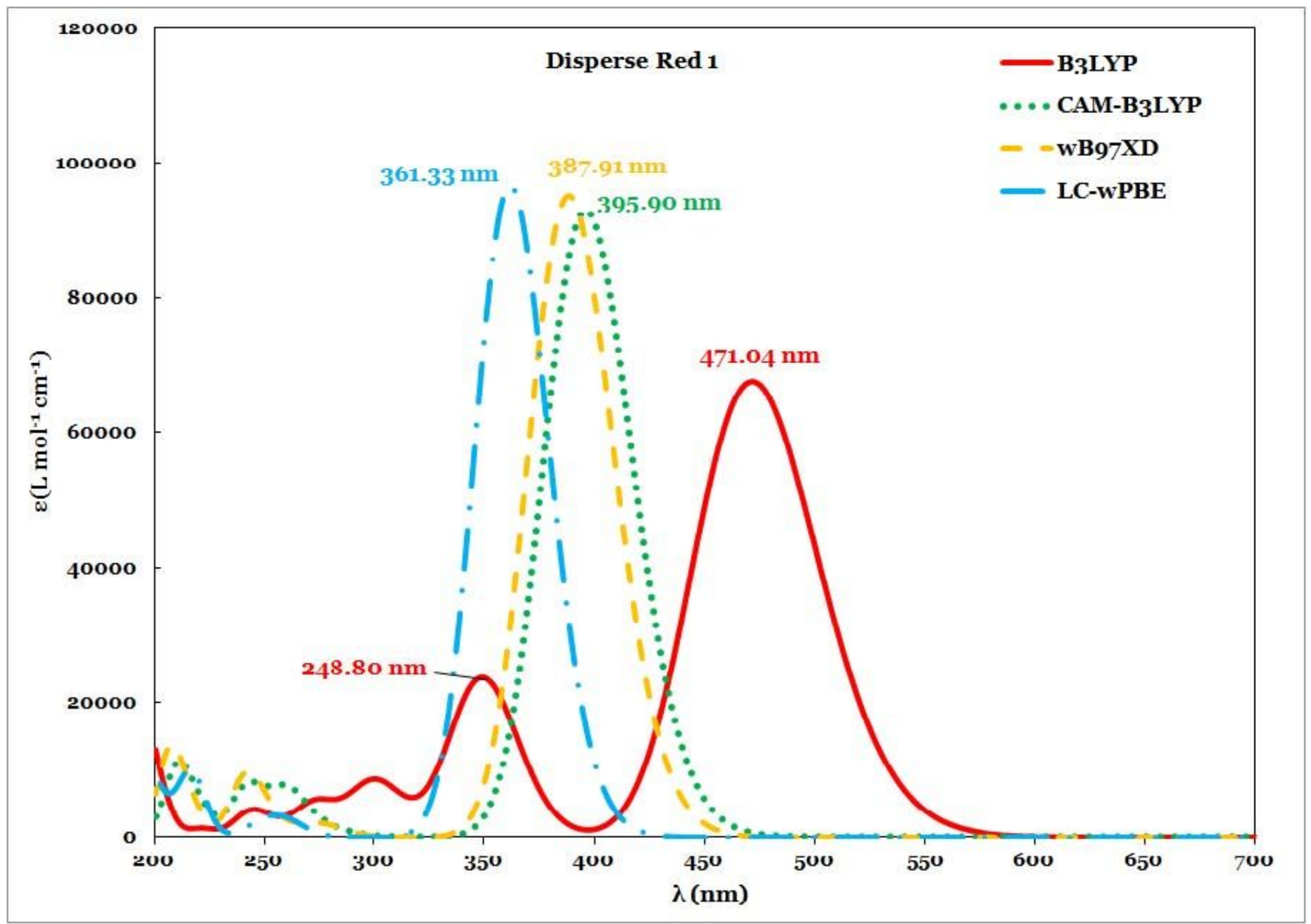

Figure 9

UV-Visible Spectra of Disperse Red 1 molecule 


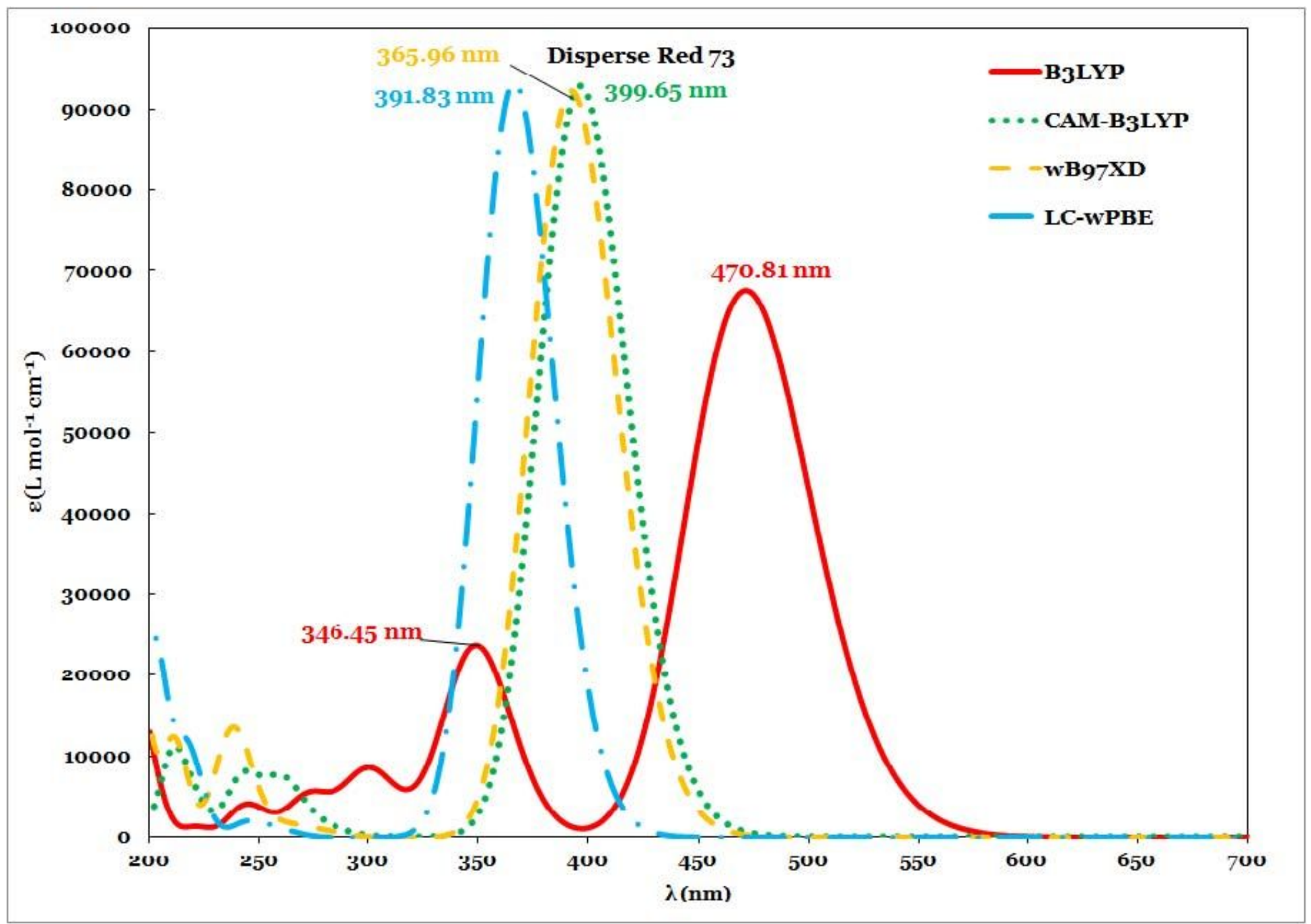

Figure 10

UV-Visible Spectra of Disperse Red 73 molecule 


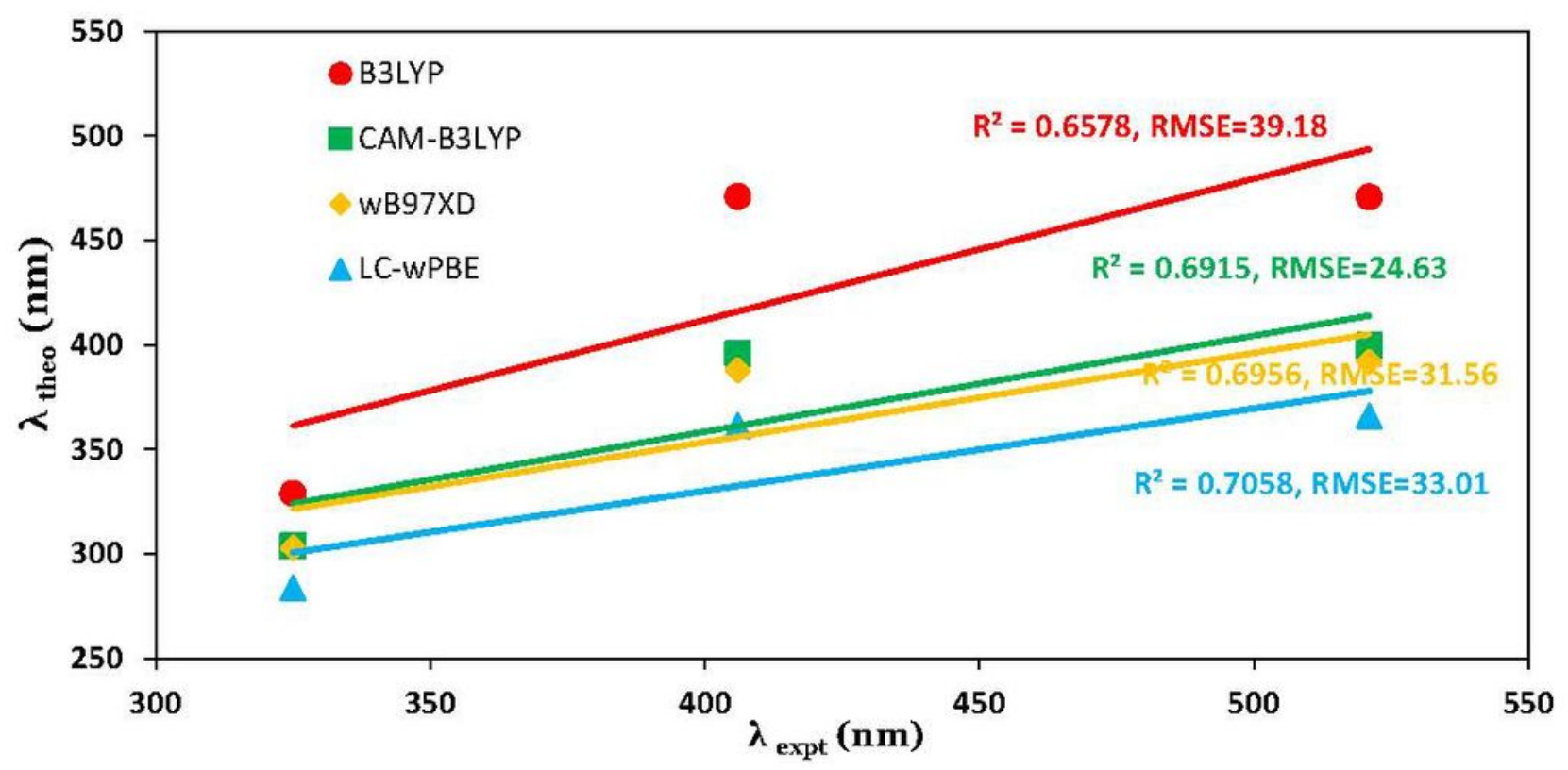

Figure 11

Comparison between theoretical ( $\lambda$ theo) and experimental wavelengths ( $\lambda$ expt) of UV-Visible spectra for all dye molecules (where R2 is the correlation coefficient and RMSE is the Root Mean Square Error)

\section{Supplementary Files}

This is a list of supplementary files associated with this preprint. Click to download.

- GraphicalAbstractREVISED.pdf 